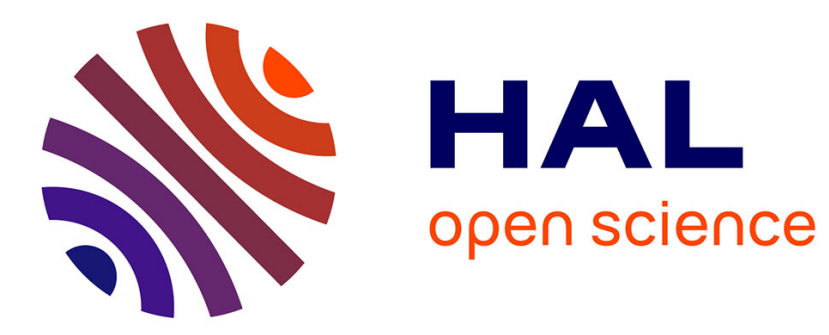

\title{
A mixed formulation for the Signorini problem in nearly incompressible elasticity
}

Faker Ben Belgacem, Yves Renard, Leila Slimane

\section{To cite this version:}

Faker Ben Belgacem, Yves Renard, Leila Slimane. A mixed formulation for the Signorini problem in nearly incompressible elasticity. Applied Numerical Mathematics, 2005, 54 (1), pp.1-22. 10.1016/j.apnum.2004.09.036 . hal-00690590

\section{HAL Id: hal-00690590 https://hal.science/hal-00690590}

Submitted on 7 Feb 2018

HAL is a multi-disciplinary open access archive for the deposit and dissemination of scientific research documents, whether they are published or not. The documents may come from teaching and research institutions in France or abroad, or from public or private research centers.
L'archive ouverte pluridisciplinaire HAL, est destinée au dépôt et à la diffusion de documents scientifiques de niveau recherche, publiés ou non, émanant des établissements d'enseignement et de recherche français ou étrangers, des laboratoires publics ou privés. 


\title{
A mixed formulation for the Signorini problem in nearly incompressible elasticity ${ }^{\text {th }}$
}

\author{
F. Ben Belgacem ${ }^{a}$, Y. Renard ${ }^{b, *}$, L. Slimane ${ }^{c}$ \\ a Mathématiques pour l'Industrie et la Physique (UMR CNRS 5640), Université Paul Sabatier, 118 route de Narbonne, \\ 31062 Toulouse cedex 04, France \\ ${ }^{\mathrm{b}}$ Mathématiques pour l'Industrie et la Physique (UMR CNRS 5640), Institut National des Sciences Appliquées, \\ 135 Avenue de Rangueil, 31077 Toulouse cedex 04, France \\ c Université de Moncton, Campus de Shippagan, 218, Boulevard J.-D. Gauthier, Shippagan, \\ Nouveau-Brunswick, E8S 1P6, Canada
}

We present a free-locking finite element approximation of the displacement-pressure formulation for the Signorini problem, in nearly incompressible elasticity. Studying the nonlinear variational problem requires an appropriate saddle point theory. An abstract framework is laid down and applied to the system of the variational inequalities we are involved in. Existence and uniqueness results for the continuous problem are proven and optimal convergence rates of the mixed Taylor-Hood finite element discretisation are proved. Some numerical experiences are reported to underline the reliability of this approach.

Keywords: Contact; Signorini problem; Quadratic finite elements; Incompressible elasticity

\footnotetext{
This work was achieved during the one-year visit of the first author to the Laboratoire Jacques-Louis Lions, (UMR CNRS 7598).

* Corresponding author.

E-mail addresses: belgacem@mip.ups-tlse.fr (F. Ben Belgacem), yves.renard@insa-toulouse.fr (Y. Renard), lslimane@umcs.ca (L. Slimane).
} 


\section{Introduction and functional tools}

In solid mechanic computations, standard low order finite element methods applied to the linear elasticity problem are known to produce unsatisfactory results when the material becomes almost incompressible, i.e., the Lamé coefficient $\lambda$ goes to infinity or, equivalently, the Poisson parameter $v$ comes close to $\frac{1}{2}$. This phenomenon, called the "numerical locking", occurs because low order Lagrange finite elements fail to approximate well divergence-free displacement fields while fulfilling the incompressibility constraint. Moreover, the stiffness matrix of the discrete problem is so ill-conditioned that computing the approximate displacement turns to be tedious. Several methods were designed to overcome the locking such as discontinuous finite elements, reduced integrations or mixed methods. We refer to the book of Brezzi and Fortin [10] where this matter is widely discussed. As explicitly noticed there, since "finite element users are more at ease with continuous discretizations", either for good or bad reasons, we choose to focus on the mixed devices for the numerical simulation of the unilateral contact Signorini problem in nearly incompressible elasticity which allow to use a large class of continuous finite elements. The major goal of the paper is to develop a theory which can be applied to the exact and the discrete problems to derive the expected error estimates.

Applying the virtual work principle to the motion equation yields the variational inequality with the displacement as the single unknown (see [13,22,12]). A low order Lagrangian finite element simulation is expected to suffer from numerical locking for high values of $\lambda$. Some computational experiences commented in the numerical section confirm such a prediction. The mixed formulation, where the pressure is considered as an independent unknown, consists in the motion variational inequality completed by the equation connecting the pressure to the displacement. The classical saddle point theory (see $[9,10]$ ), cannot be applied to this problem, due to the contact nonlinearity. It is then necessary to write down a saddle point theory well suited to the Signorini system. This is the core of Section 3 where existence and uniqueness results are stated with standard coerciveness and continuity assumptions on the bilinear forms involved in the variational problem while the proof of the uniform stability with respect to $\lambda$ is derived from an inf-sup condition. Then, comes the description of the Galerkin discretization which is very often nonconforming. To tackle the question of the error estimation we need to make the same hypothesis as for the continuous case, in particular we assume the compatibility of the discrete displacement and the pressure spaces regarding the inf-sup condition, and putting together the mathematical tools employed by Brezzi in [9] and those used by Falk in [15], we are able to derive an abstract result that turns to be useful for the finite element approximation of the saddle point Signorini problem. This is realized in Section 4 where the Taylor-Hood mixed elements are chosen. Applying the abstract framework provides the expected optimal converge rate free of locking (the estimate does not depend on $\lambda$ ) for raisonable regularity on both the displacement and the pressure. Section 5 is dedicated to a numerical discussion in order to highlight the reliability of the mixed approximation of the Signorini problem.

Notation. We need to set some notation and to recall some functional tools necessary to our analysis. Let $\Omega \subset \mathbb{R}^{2}$ be a Lipschitz domain, the generic point of $\Omega$ is denoted $\mathbf{x}$. The Lebesgue space $L^{2}(\Omega)$ is endowed with the norm

$$
\|\psi\|_{L^{2}(\Omega)}=\left(\int_{\Omega}|\psi(\mathbf{x})|^{2} \mathrm{~d} \mathbf{x}\right)^{1 / 2}
$$


and $L_{0}^{2}(\Omega)=L^{2}(\Omega) / \mathbb{R}$ is the closed subspace containing the null-averaged functions. We use the standard Sobolev space $H^{m}(\Omega), m \geqslant 1$, provided with the norm

$$
\|\psi\|_{H^{m}(\Omega)}=\left(\sum_{0 \leqslant|\alpha| \leqslant m}\left\|\partial^{\alpha} \psi\right\|_{L^{2}(\Omega)}^{2}\right)^{1 / 2},
$$

where $\alpha=\left(\alpha_{1}, \alpha_{2}\right)$ is a multi-index in $\mathbb{N}^{2}$ and the symbol $\partial^{\alpha}$ represents a partial derivative $\left(H^{0}(\Omega)=\right.$ $\left.L^{2}(\Omega)\right)$. The fractional order Sobolev space $H^{v}(\Omega), v \in \mathbb{R}^{+} \backslash \mathbb{N}$, is defined by the norm

$$
\|\psi\|_{H^{\nu}(\Omega)}=\left(\|\psi\|_{H^{m}(\Omega)}^{2}+\sum_{|\alpha|=m} \int_{\Omega} \int_{\Omega} \frac{\left(\partial^{\alpha} \psi(\mathbf{x})-\partial^{\alpha} \psi(\mathbf{y})\right)^{2}}{|\mathbf{x}-\mathbf{y}|^{2+2 \theta}} \mathrm{d} \mathbf{x} \mathrm{d} \mathbf{y}\right)^{1 / 2},
$$

where $v=m+\theta, m$ is the integer part of $v$ and $\theta \in] 0,1[$ is the decimal part (see $[1,18]$ ). The closure in $H^{v}(\Omega)$ of $\mathscr{D}(\Omega)$ is denoted $H_{0}^{v}(\Omega)$, where $\mathscr{D}(\Omega)$ is the space of infinitely differentiable functions whose support is contained in $\Omega$. For a given portion $\gamma$ of the boundary $\partial \Omega$, the space $H_{0}^{1}(\Omega, \gamma)$ is made of the functions that vanish on $\gamma$. Let $v$ be any positive real number, the Hilbert space $H^{v}(\gamma)$ is defined as the range of $H^{v+1 / 2}(\Omega)$ by the trace operator, it is then endowed by the image norm

$$
\|\psi\|_{H^{\nu}(\gamma)}=\inf _{\chi \in H^{v+1 / 2}(\Omega), \chi \mid \gamma=\psi}\|\chi\|_{H^{\nu+1 / 2}(\Omega)} .
$$

When $\gamma$ is sufficiently regular, it is possible to write down an explicit norm of $H^{v}(\gamma)$, while for polygonal lines, which is currently the situation, it turns out to be more complicated to have an explicit norm, especially for $v \geqslant \frac{1}{2}$. The space $H^{v}(\gamma)^{\prime}$ stands for the topological dual space of $H^{v}(\gamma)$ and the duality pairing is denoted by $\langle\cdot, \cdot\rangle_{\nu, \gamma}$. The special space $H_{00}^{1 / 2}(\gamma)$ is defined as the set of the restrictions to $\gamma$ of the functions of $H^{1 / 2}(\partial \Omega)$ that vanish on $\partial \Omega \backslash \gamma$, it is also obtained by Hilbertian interpolation between $H_{0}^{1}(\gamma)$ and $L^{2}(\gamma)($ see $[1,23])$.

\section{The Hermann mixed formulation of the Signorini problem}

Let $\Omega$ be an arbitrary Lipschitz domain in $\mathbb{R}^{2}$, the boundary $\partial \Omega$ being a union of two nonoverlapping portions $\Gamma_{\mathbf{u}}$ and $\Gamma_{C}$ and let $\left\{\mathbf{c}_{1}, \mathbf{c}_{2}\right\}$ be the common vertices of $\Gamma_{C}$ and $\Gamma_{\mathbf{u}}$. Denote by $\mathbf{n}$ the outward unit normal to $\partial \Omega$. In structural mechanics, the displacement of an elastic body $\Omega$ (represented in Fig. 1) supported by a frictionless rigid foundation, fixed along $\Gamma_{\mathbf{u}}$ and subjected to external forces $\mathbf{f}_{\mid \Omega} \in L^{2}(\Omega)^{2}$ satisfies the following partial differential equation

$$
\begin{aligned}
& -\operatorname{div} \sigma(\mathbf{u})=\mathbf{f} \text { in } \Omega, \\
& \mathbf{u}=\mathbf{u}_{D} \quad \text { on } \Gamma_{\mathbf{u}},
\end{aligned}
$$

where $\mathbf{u}_{D}$ is a prescribed displacement. The bold symbol div stands for the divergence operator of a tensor function and is defined as $\operatorname{div} \sigma=\left(\frac{\partial \sigma_{i j}}{\partial x_{j}}\right)_{i}$. To close the system, frictionless contact conditions are needed on $\Gamma_{C}$. Denoting $\sigma_{\mathbf{n}}$ the normal component of the stress force $(\boldsymbol{\sigma} \mathbf{n})$ and $\boldsymbol{\sigma}_{\mathbf{t}}$ its tangential component such that $\boldsymbol{\sigma} \mathbf{n}=\sigma_{\mathbf{n}} \mathbf{n}+\sigma_{\mathbf{t}}$, the contact conditions are formulated as follows (see [13,20,22]):

$$
\begin{aligned}
& \mathbf{u} \cdot \mathbf{n} \leqslant 0, \quad \sigma_{\mathbf{n}} \leqslant 0, \quad \sigma_{\mathbf{n}}(\mathbf{u} \cdot \mathbf{n})=0, \\
& \boldsymbol{\sigma}_{\mathbf{t}}=0 .
\end{aligned}
$$




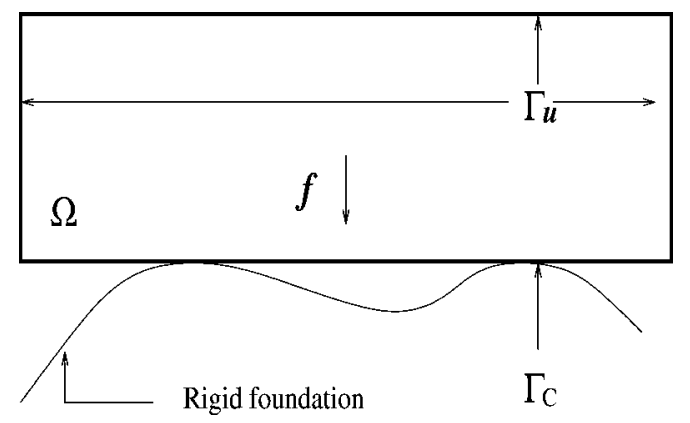

Fig. 1. The configuration of the elastic solid $\Omega$ before deformation subjected, e.g., to its weight. The bottom edge $\Gamma_{C}$ is the region candidate to be in contact with the rigid foundation. The effective contact zone is not known before calculations.

The stress tensor is obtained from the displacement through the Hook constitutive law

$$
\boldsymbol{\sigma}(\mathbf{u})=2 \mu \boldsymbol{\varepsilon}(\mathbf{u})+\lambda(\operatorname{div} \mathbf{u}) I,
$$

where under small perturbations hypothesis, $\boldsymbol{\varepsilon}(\mathbf{u})$ is the linear strain tensor; i.e., $\boldsymbol{\varepsilon}(\mathbf{u})=\frac{1}{2}\left(\nabla \mathbf{u}+\nabla \mathbf{u}^{\mathrm{T}}\right)$, $I$ is the metric tensor and $(\lambda, \mu)$ are the Lamé parameters. Then, the static equilibrium equation (2.1) can be rewritten as follows

$$
-2 \mu(\operatorname{div} \boldsymbol{\varepsilon}(\mathbf{u})-\lambda \nabla(\operatorname{div} \mathbf{u})=\mathbf{f} \quad \text { in } \Omega .
$$

Only for simplification we shall assume in the subsequent discussion that $\mathbf{u}_{D}=0$. As $\Gamma_{\mathbf{u}}=\partial \Omega$ (and $\Gamma_{C}=\emptyset$ ), we are therefore in the linear context, near the incompressibility limit $\lambda \rightarrow+\infty$, the finite element discretization of the variational form of problem (2.2)-(2.4) suffers from the "numerical locking" phenomenon; there can be a decrease in the accuracy of the computed displacement. This is particularly observed as lower degree finite elements are used (of degree $\leqslant 3$ ). The reason why such a phenomenon occurs is the inability of the discrete space to approximate accurately $\mathbf{u}$ while satisfying the incompressibility ( $\operatorname{div} \mathbf{u}=0)$. The numerical locking is highlighted in many works among which we recommend $[3,28]$. To avoid this phenomenon (see [10]), the most popular strategy is to resort to the mixed formulation of the problem (2.2)-(2.4). This allows us to reduce the severity of the constraint ( $\operatorname{div} \mathbf{u}=0$ ) by enforcing it only weakly. Recall that, when $\Gamma_{\mathbf{u}}=\partial \Omega$ the technique consists to introduce the auxiliary pressure $(\lambda \operatorname{div} \mathbf{u})$ as an independent unknown $\left(\lambda(\operatorname{div} \mathbf{u}) \in L_{0}^{2}(\Omega)\right)$, the pressure has a zero mean value because of the Dirichlet condition. Unfortunately, for some reasons that will appear later this choice is not appropriate for our Signorini problem. The convenient way to proceed is to split the pressure $\lambda(\operatorname{div} \mathbf{u})$ into

$$
\lambda(\operatorname{div} \mathbf{u})=p+\frac{\lambda}{|\Omega|}\left(\int_{\Omega} \operatorname{div} \mathbf{u} \mathrm{d} \mathbf{x}\right),
$$

with $p \in L_{0}^{2}(\Omega)$ and to write down a mixed variational inequality on the displacement-pressure $(\mathbf{u}, p)$ unknowns. First, let us describe the suitable functional framework to handle the nearly incompressible Signorini problem. The displacements belong to $H_{0}^{1}\left(\Omega, \Gamma_{\mathbf{u}}\right)^{2}$. The unilateral contact condition on $\Gamma_{C}$ is weakly formulated by means of the closed convex cone

$$
\left.\mathbf{K}(\Omega)=\mathbf{v} \in H_{0}^{1}\left(\Omega, \Gamma_{\mathbf{u}}\right)^{2},(\mathbf{v} \cdot \mathbf{n})_{\mid \Gamma_{C}} \leqslant 0, \text { a.e. }\right\} .
$$


The Hermann weak formulation adapted to the Signorini problem leads to a variational system that reads as: find $(\mathbf{u}, p) \in \mathbf{K}(\Omega) \times L_{0}^{2}(\Omega)$ such that

$$
\begin{aligned}
& a_{\lambda}(\mathbf{u}, \mathbf{v}-\mathbf{u})+b(\mathbf{v}-\mathbf{u}, p) \geqslant(\mathbf{f}, \mathbf{v}-\mathbf{u})_{L^{2}(\Omega)} \quad \forall \mathbf{v} \in \mathbf{K}(\Omega), \\
& b(\mathbf{u}, q)-\frac{1}{\lambda}(p, q)_{L^{2}(\Omega)}=0 \quad \forall q \in L_{0}^{2}(\Omega) .
\end{aligned}
$$

In (2.6), (2.7) we have set: $\forall \mathbf{u}, \mathbf{v} \in H_{0}^{1}\left(\Omega, \Gamma_{\mathbf{u}}\right)^{2}, \forall q \in L_{0}^{2}(\Omega)$,

$$
\begin{aligned}
& a_{\lambda}(\mathbf{u}, \mathbf{v})=2 \mu\left(\boldsymbol{\varepsilon}(\mathbf{u}), \boldsymbol{\varepsilon}(\mathbf{v}){ }_{L^{2}(\Omega)^{4}}+\frac{\lambda}{|\Omega|}\left(\int_{\Omega} \operatorname{div} \mathbf{u} \mathrm{d} \mathbf{x}\right)\left(\int_{\Omega} \operatorname{div} \mathbf{v} \mathrm{d} \mathbf{x}\right),\right. \\
& b(\mathbf{v}, q)=\int_{\Omega}(\operatorname{div} \mathbf{v}) q \mathrm{~d} \mathbf{x} .
\end{aligned}
$$

Taking into account the term in $\lambda$ in the expression of $a_{\lambda}(\cdot, \cdot)$ can be viewed as an augmented Lagrangian procedure (see [17, Chapter 4]). The proof of the equivalence between (2.6) and (2.4) at one side and between (2.7) and (2.5) at the other side is skipped over as no particular technical difficulty arises.

Remark 2.1. The normal component $\sigma_{\mathbf{n}}$ of the normal stress force is given by

$$
\sigma_{\mathbf{n}}=\left(2 \mu \boldsymbol{\varepsilon}(\mathbf{u}) \mathbf{n} \cdot \mathbf{n}+p+\frac{\lambda}{|\Omega|} \int_{\Omega} \operatorname{div} \mathbf{u} \mathrm{d} \mathbf{x} .\right.
$$

For convenience (see Section 4 ) this normal stress will be denoted by $\sigma_{\mathbf{n}}^{\lambda}$, to underline the dependency on $\lambda$, while $\sigma_{\mathbf{n}}=(2 \mu \boldsymbol{\varepsilon}(\mathbf{u}) \mathbf{n} \cdot \mathbf{n}+p)$ stands only for the part that is independent of $\lambda$. Moreover, in the variational formulation (2.6), (2.7), the mathematical sense given to condition (2.3) is the following

$$
\begin{aligned}
& \left\langle\boldsymbol{\sigma}^{\lambda}(\mathbf{u}) \mathbf{n}, \mathbf{v}\right\rangle_{1 / 2, \partial \Omega} \geqslant 0, \quad \forall \mathbf{v} \in H_{00}^{1 / 2}\left(\partial \Omega, \Gamma_{\mathbf{u}}\right)^{2},(\mathbf{v} \cdot \mathbf{n})_{\mid \Gamma_{C}} \leqslant 0, \\
& \left\langle\boldsymbol{\sigma}^{\lambda}(\mathbf{u}) \mathbf{n}, \mathbf{u}_{1 / 2, \partial \Omega}=0,\right.
\end{aligned}
$$

where $H_{00}^{1 / 2}\left(\partial \Omega, \Gamma_{\mathbf{u}}\right)^{2}$ is the subspace of $H^{1 / 2}(\partial \Omega)^{2}$ of the functions $\mathbf{v}$ whose normal component $(\mathbf{v} \cdot \mathbf{n})$ vanishes on $\Gamma_{\mathbf{u}}$. Roughly, (2.9) says that $\sigma_{\mathbf{t}}=0$ and $\sigma_{\mathbf{n}}^{\lambda} \leqslant 0$ on $\Gamma_{C}$ while (2.10) expresses the exclusion (in mechanic terminology) or the complementarity (in optimization terminology) condition $\sigma_{\mathbf{n}}^{\lambda}(\mathbf{u} \cdot \mathbf{n})=0$ on $\Gamma_{C}$.

Remark 2.2. Most often, a part of the boundary of the domain $\Omega$ is subjected to boundary forces which correspond to Neumann conditions. However, in this work, we consider only Dirichlet and unilateral contact conditions which is the "worst case" with respect to the stability and the convergence of the methods to be studied.

\section{An abstract problem}

Let us now consider an abstract problem that will be successfully applied to the variational problem (2.6), (2.7) and its finite element approximation. This framework is readily extended to more general saddle point problems for a class of variational inequalities, we refer to the work of [27] (see also [26]). 


\subsection{Continuous setting of a mixed variational inequality}

Let $\mathbf{X}$ and $M$ be two Hilbert spaces with inner products $(\cdot, \cdot)_{\mathbf{X}}$ and $(\cdot, \cdot)_{M}$; the associated norms being $\|\cdot\|_{\mathbf{X}}$ and $\|\cdot\|_{M}$, respectively. Denote by $\mathbf{X}^{\prime}$ and $M^{\prime}$ their dual spaces. Consider two continuous bilinear forms $a_{0}(\cdot, \cdot)$ and $a_{1}(\cdot, \cdot)$ on $\mathbf{X}$ and set, for any $\varepsilon>0, a_{\varepsilon}(\cdot, \cdot)=a_{0}(\cdot, \cdot)+\frac{1}{\varepsilon} a_{1}(\cdot, \cdot)$. Let $c_{\varepsilon}(\cdot, \cdot)$ be a bilinear form on $M$ and $b(\cdot, \cdot)$ a continuous bilinear form on $\mathbf{X} \times M$. Denote by $\mathbf{K}$ a closed convex cone of $\mathbf{X}$ with vertex at 0 . Then, for a given $\ell \in \mathbf{X}^{\prime}$ and $\chi \in M^{\prime}$, we investigate the variational problem: find $(\mathbf{u}, p) \in \mathbf{K} \times M$ such that

$$
\begin{aligned}
& a_{\varepsilon}(\mathbf{u}, \mathbf{v}-\mathbf{u})+b(\mathbf{v}-\mathbf{u}, p) \geqslant \ell(\mathbf{v}-\mathbf{u}) \quad \forall \mathbf{v} \in \mathbf{K}, \\
& b(\mathbf{u}, q)-c_{\varepsilon}(p, q)=\chi(q) \quad \forall q \in M .
\end{aligned}
$$

In [19], a study of a different version of the mixed problem (3.1), (3.2) can be found for which $\varepsilon=0$ and a frictional nondifferentiable term is added to (3.1). Existence and stability results are proven there. This problem can be reformulated in the symmetric case as a saddle point problem by considering the Lagrangian functional, $\forall \mathbf{v} \in \mathbf{X}, \forall q \in M$,

$$
\mathcal{L}(\mathbf{v}, q)=\frac{1}{2} a_{\varepsilon}(\mathbf{v}, \mathbf{v})+b(\mathbf{v}, q)-\frac{1}{2} c_{\varepsilon}(q, q)-\ell(\mathbf{v})-\chi(q),
$$

and $(\mathbf{u}, p)$ is then characterized as the saddle point of $\mathcal{L}$,

$$
\mathcal{L}(\mathbf{u}, p)=\inf _{\mathbf{v} \in \mathbf{K}} \sup _{q \in M} \mathcal{L}(\mathbf{v}, q)=\sup _{q \in M} \inf _{\mathbf{v} \in \mathbf{K}} \mathcal{L}(\mathbf{v}, q) .
$$

In order to state existence and uniqueness results we need some additional hypothesis which are currently made for the saddle-point theory even in the linear context.

(i) The bilinear form $a_{0}(\cdot, \cdot)$ is coercive and the form $a_{1}(\cdot, \cdot)$ is positive semi-definite

$$
\begin{array}{ll}
a_{0}(\mathbf{v}, \mathbf{v}) \geqslant \alpha\|\mathbf{v}\|_{\mathbf{X}}^{2}, & \forall \mathbf{v} \in \mathbf{X}, \\
a_{1}(\mathbf{v}, \mathbf{v}) \geqslant 0, & \forall \mathbf{v} \in \mathbf{X} .
\end{array}
$$

Notice that a direct consequence is that the form $a_{\varepsilon}(\cdot, \cdot)$ is also elliptic because $a_{\varepsilon}(\cdot, \cdot) \geqslant a_{0}(\cdot, \cdot)$.

(ii) There exists two constants $\gamma$ and $\tilde{\gamma}$ such that: $\forall p, q \in M$,

$$
c_{\varepsilon}(p, q) \leqslant \tilde{\gamma} \varepsilon\|p\|_{M}\|q\|_{M}, \quad c_{\varepsilon}(q, q) \geqslant \gamma \varepsilon\|q\|_{M}^{2} .
$$

Proposition 3.1. Assume that hypothesis (i)-(ii) hold. Then, problem (3.1), (3.2) has only one solution $(\mathbf{u}, p) \in \mathbf{K} \times M$ that satisfies

$$
\alpha\|\mathbf{u}\|_{\mathbf{X}}+\varepsilon \gamma\|p\|_{M} \leqslant \frac{1}{\alpha}\|\ell\|_{\mathbf{X}^{\prime}}+\frac{1}{\gamma}\|\chi\|_{M^{\prime}} .
$$

Proof. Let us rewrite the mixed system (3.1), (3.2) as a standard variational inequality. Denote by $\mathcal{X}$ the Hilbert space $\mathbf{X} \times M$ endowed with the natural norm, $\mathcal{K} \subset \mathcal{X}$ stands for the closed convex cone $\mathbf{K} \times M$ and $\mathcal{A}_{\varepsilon}$ and $\mathcal{L}$ are the bilinear and linear forms respectively defined by: $\forall \mathbf{u}^{*}=(\mathbf{u}, p), \mathbf{v}^{*}=(\mathbf{v}, q) \in \mathcal{X}$,

$$
\begin{aligned}
& \mathcal{A}_{\varepsilon}\left(\mathbf{u}^{*}, \mathbf{v}^{*}=a_{\varepsilon}(\mathbf{u}, \mathbf{v})+b(\mathbf{v}, p)-b(\mathbf{u}, q)+c_{\varepsilon}(p, q),\right. \\
& \mathcal{L}\left(\mathbf{v}^{*}=\ell(\mathbf{v})-\chi(q) .\right.
\end{aligned}
$$


Then, it is easily checked that $\mathbf{u}^{*}=(\mathbf{u}, p)$ is solution of (3.1), (3.2) if and only if it is solution of the variational inequality: Find $\mathbf{u}^{*}=(\mathbf{u}, p) \in \mathcal{K}$ such that

$$
\mathcal{A}_{\varepsilon}\left(\mathbf{u}^{*}, \mathbf{v}^{*}-\mathbf{u}^{*} \geqslant \mathcal{L}\left(\mathbf{v}^{*}-\mathbf{u}^{*}, \quad \forall \mathbf{v}^{*} \in \mathcal{K} .\right.\right.
$$

The form $\mathcal{A}_{\varepsilon}$ being continuous and positive definite (with an ellipticity constant dependent on $\varepsilon$ ) and the linear form $\mathcal{L}$ is continuous then applying Stampachia theorem to the variational inequality (3.3), the mixed problem (3.1), (3.2), has only one solution $\mathbf{u}^{*}=(\mathbf{u}, p) \in \mathcal{K}$. For the stability, taking $\mathbf{v}^{*}=2 \mathbf{u}^{*}$ and $\mathbf{v}^{*}=0$ in (3.3) yields

$$
a_{\varepsilon}(\mathbf{u}, \mathbf{u})+c_{\varepsilon}(p, p)=\ell(\mathbf{u})-\chi(p)
$$

The coerciveness of $a_{\varepsilon}(\cdot, \cdot)$ and $c_{\varepsilon}(\cdot, \cdot)$ and Young inequality complete the proof.

The estimate derived on $\mathbf{u}$ looks satisfactory, in the contrary that proven on $p$ does not. Indeed, for small $\varepsilon$, which is the case of our interest, we have not a uniform bound on $\|p\|_{M}$. However, if we make an additional assumption on $b(\cdot, \cdot)$ it is possible to recover the optimality. Let us first introduce the subspace $\mathbf{W}$ of $\mathbf{X}$

$$
\mathbf{W}=\mathbf{K} \cap(-\mathbf{K})=\{\mathbf{v} \in \mathbf{K},-\mathbf{v} \in \mathbf{K}\},
$$

and assume that

(iii) There exists a constant $\beta>0$ such that the following inf-sup condition holds

$$
\inf _{q \in M} \sup _{\mathbf{v} \in \mathbf{W}} \frac{b(\mathbf{v}, q)}{\|\mathbf{v}\|_{\mathbf{X}}\|q\|_{M}} \geqslant \beta \text {. }
$$

(iv) The form $a_{1}(\cdot, \cdot)$ vanishes on $\mathbf{W}$ meaning that:

$$
a_{1}(\mathbf{u}, \mathbf{v})=0, \quad \forall \mathbf{u} \in \mathbf{X}, \forall \mathbf{v} \in \mathbf{W} .
$$

An immediate consequence of the definition of $\mathbf{W}$ and of the assumption (iv) is that

$$
a_{0}(\mathbf{u}, \mathbf{v})+b(\mathbf{v}, p)=a_{\varepsilon}(\mathbf{u}, \mathbf{v})+b(\mathbf{v}, p)=\ell(\mathbf{v}), \quad \forall \mathbf{v} \in \mathbf{W} .
$$

Theorem 3.2. Assume that hypothesis (i)-(iv) hold. Then, the mixed problem (3.1), (3.2) has only one solution $(\mathbf{u}, p) \in \mathbf{K} \times M$ that satisfies

$$
\|\mathbf{u}\|_{\mathbf{X}}+\beta\|p\|_{M} \leqslant C\left(\|\ell\|_{\mathbf{X}^{\prime}}+\|\chi\|_{M^{\prime}} .\right.
$$

The constant $C$ is independent of $\varepsilon$.

Proof. The inf-sup condition and the variational identity (3.5) give

$$
\beta\|p\|_{M} \leqslant \sup _{\mathbf{v} \in \mathbf{W}} \frac{b(\mathbf{v}, p)}{\|\mathbf{v}\|_{\mathbf{X}}}=\sup _{\mathbf{v} \in \mathbf{W}} \frac{a_{0}(\mathbf{u}, \mathbf{v})-\ell(\mathbf{v})}{|\mathbf{v}|_{\mathbf{X}}} .
$$

We complete thanks to the uniform stability on $\mathbf{u}$ of Proposition 3.1 .

Remark 3.1. From (3.4) we can derive that

$$
\frac{1}{\varepsilon} a_{1}(\mathbf{u}, \mathbf{u}) \leqslant \ell(\mathbf{u})-\chi(p),
$$

form which we obtain thanks to Theorem 3.2

$$
\overline{a_{1}(\mathbf{u}, \mathbf{u})} \leqslant C \sqrt{\varepsilon}\left(\|\ell\|_{\mathbf{X}^{\prime}}+|\chi|_{M^{\prime}} .\right.
$$




\subsection{Ritz-Galerkin approximation and an error estimate}

Let $\mathbf{X}_{h}$ and $M_{h}$ be two finite dimensional sub-spaces which are supposed to be appropriate internal approximations of $\mathbf{X}$ and $M$ respectively, for small values of the discretization parameter $h$. Introduce a closed convex cone $\mathbf{K}_{h}$ of $\mathbf{X}_{h}$ with vertex 0 that is not necessarily contained in $\mathbf{K}\left(\mathbf{K}_{h} \not \subset \mathbf{K}\right)$ and consider the nonconforming Riesz-Galerkin approximation of the continuous variational problem (3.1), (3.2): find $\left(\mathbf{u}_{h}, p_{h}\right) \in \mathbf{K}_{h} \times M_{h}$ such that

$$
\begin{aligned}
& a_{\varepsilon}\left(\mathbf{u}_{h}, \mathbf{v}_{h}-\mathbf{u}_{h}\right)+b\left(\mathbf{v}_{h}-\mathbf{u}_{h}, p_{h}\right) \geqslant \ell\left(\mathbf{v}_{h}-\mathbf{u}_{h}\right) \quad \forall \mathbf{v}_{h} \in \mathbf{K}_{h}, \\
& b\left(\mathbf{u}_{h}, q_{h}\right)-c_{\varepsilon}\left(p_{h}, q_{h}\right)=\chi\left(q_{h}\right) \quad \forall q_{h} \in M_{h} .
\end{aligned}
$$

For the complete analysis of this system and in order to derive estimates that do not depend on $\varepsilon$ we need to modify hypothesis (iii) and (iv) to render them well adapted to the discrete framework. Denote $\mathbf{W}_{h}=\mathbf{K}_{h} \cap\left(-\mathbf{K}_{h}\right)$ and let us make the following hypothesis

(iii) $h$ There exists a constant $\tilde{\beta}>0$ independent of $h$ such that the following inf-sup condition holds

$$
\inf _{q_{h} \in M_{h}} \sup _{\mathbf{v}_{h} \in \mathbf{W}_{h}} \frac{b\left(\mathbf{v}_{h}, q_{h}\right)}{\left\|\mathbf{v}_{h}\right\|_{\mathbf{X}}\left\|q_{h}\right\|_{M}} \geqslant \tilde{\beta} .
$$

(iv) ${ }_{h}$ We have $\mathbf{W}_{h} \subset \mathbf{W}$.

In the same way as in (3.5) a direct consequence of the hypothesis (iv) $h$ is that

$$
a_{0}\left(\mathbf{u}_{h}, \mathbf{v}_{h}\right)+b\left(\mathbf{v}_{h}, p_{h}\right)=\ell\left(\mathbf{v}_{h}\right), \quad \forall \mathbf{v}_{h} \in \mathbf{W}_{h},
$$

which is necessary, together with the inf-sup condition, for the uniform stabilility of the discrete pressure.

Theorem 3.3. Assume that the hypotheses (i)-(ii) and (iii) ${ }_{h}-(\mathrm{iv})_{h}$ hold. Then, the discrete mixed problem (3.6), (3.7) has only one solution $\left(\mathbf{u}_{h}, p_{h}\right) \in \mathbf{K}_{h} \times M_{h}$ such that

$$
\left\|\mathbf{u}_{h}\right\|_{\mathbf{x}}+\tilde{\beta}\left\|p_{h}\right\|_{M} \leqslant C\left(\|\ell\|_{\mathbf{X}^{\prime}}+\|\chi\|_{M^{\prime}} .\right.
$$

Moreover, we have the following error estimate:

$$
\begin{aligned}
& \left\|\mathbf{u}-\mathbf{u}_{h}\right\|_{\mathbf{X}}^{2}+\left\|p-p_{h}\right\|_{M}^{2} \\
& \quad \leqslant C\left(\inf _{\substack{\mathbf{v}_{h} \in \mathbf{K}_{h} \\
a_{1}\left(\mathbf{u}-\mathbf{v}_{h}, \mathbf{u}-\mathbf{v}_{h}\right)=0}}\left[\left\|\mathbf{u}-\mathbf{v}_{h}\right\|_{\mathbf{X}}^{2}+e i_{\varepsilon}\left(\mathbf{v}_{h}\right)\right]+\inf _{\mathbf{v} \in \mathbf{K}} e c_{\varepsilon}(\mathbf{v})+\inf _{q_{h} \in M_{h}}\left\|p-q_{h}\right\|_{M}^{2}\right) .
\end{aligned}
$$

The constant $C$ does not depend on $\varepsilon$ nor on $h$ and where we have set

$$
\begin{aligned}
& e i_{\varepsilon}\left(\mathbf{v}_{h}\right)=a_{\varepsilon}\left(\mathbf{u}, \mathbf{v}_{h}-\mathbf{u}\right)+b\left(\mathbf{v}_{h}-\mathbf{u}, p\right)-\ell\left(\mathbf{v}_{h}-\mathbf{u}\right), \\
& e c_{\varepsilon}(\mathbf{v})=a_{\varepsilon}\left(\mathbf{u}, \mathbf{v}-\mathbf{u}_{h}\right)+b\left(\mathbf{v}-\mathbf{u}_{h}, p\right)-\ell\left(\mathbf{v}-\mathbf{u}_{h}\right) .
\end{aligned}
$$

Proof. Following the same line as in the proof of Theorem 3.2 we obtain the existence, the uniqueness and the stability result. In order to state the error estimate (3.8) let $\mathbf{v}_{h} \in \mathbf{W}_{h}$ and $q_{h} \in M_{h}$ be arbitrarily chosen, then we have

$$
\begin{aligned}
b\left(\mathbf{v}_{h}, q_{h}-p_{h}\right) & =b\left(\mathbf{v}_{h}, q_{h}\right)-b\left(\mathbf{v}_{h}, p_{h}\right)=b\left(\mathbf{v}_{h}, q_{h}\right)+a_{\varepsilon}\left(\mathbf{u}_{h}, \mathbf{v}_{h}\right)-\ell\left(\mathbf{v}_{h}\right) \\
& =b\left(\mathbf{v}_{h}, q_{h}-p\right)+a_{\varepsilon}\left(\mathbf{u}_{h}-\mathbf{u}, \mathbf{v}_{h}\right)=b\left(\mathbf{v}_{h}, q_{h}-p\right)+a_{0}\left(\mathbf{u}_{h}-\mathbf{u}, \mathbf{v}_{h}\right) .
\end{aligned}
$$


In view of the inf-sup condition of assumption (iii) ${ }_{h}$ it comes out that: $\forall q_{h} \in M_{h}$

$$
\left\|p-p_{h}\right\|_{M} \leqslant \eta\left(\left\|\mathbf{u}-\mathbf{u}_{h}\right\|_{\mathbf{x}}+\left|p-q_{h}\right|_{M} .\right.
$$

Next, notice that the discrete mixed problem (3.6), (3.7) can also be reformulated in the same way as the variational inequality (3.3). Then, we derive that: $\forall \mathbf{v}^{*} \in \mathcal{K}, \forall \mathbf{v}_{h}^{*} \in \mathcal{K}_{h}$,

$$
\begin{aligned}
\mathcal{A}_{\varepsilon}\left(\mathbf{u}^{*}-\mathbf{u}_{h}^{*}, \mathbf{u}^{*}-\mathbf{u}_{h}^{*}\right)= & \mathcal{A}_{\varepsilon}\left(\mathbf{u}^{*}, \mathbf{u}^{*}\right)-\mathcal{A}_{\varepsilon}\left(\mathbf{u}^{*}, \mathbf{u}_{h}^{*}\right)-\mathcal{A}_{\varepsilon}\left(\mathbf{u}_{h}^{*}, \mathbf{u}^{*}+\mathcal{A}_{\varepsilon}\left(\mathbf{u}_{h}^{*}, \mathbf{u}_{h}^{*}\right.\right. \\
= & \mathcal{L}\left(\mathbf{u}^{*}-\mathbf{v}_{h}^{*}-\mathcal{A}_{\varepsilon}\left(\mathbf{u}^{*}, \mathbf{u}^{*}-\mathbf{v}_{h}^{*}\right.\right. \\
& +\mathcal{L}\left(\mathbf{u}_{h}^{*}-\mathbf{v}^{*}-\mathcal{A}_{\varepsilon}\left(\mathbf{u}^{*}, \mathbf{u}_{h}^{*}-\mathbf{v}^{*}+\mathcal{A}_{\varepsilon}\left(\mathbf{u}^{*}-\mathbf{u}_{h}^{*}, \mathbf{u}^{*}-\mathbf{v}_{h}^{*} .\right.\right.\right.
\end{aligned}
$$

After obvious simplifications we obtain: $\forall \delta, \delta^{\prime}>0, \forall \mathbf{v}^{*} \in \mathcal{K}, \forall \mathbf{v}_{h}^{*} \in \mathcal{K}_{h}$,

$$
\begin{aligned}
\mathcal{A}_{\varepsilon}\left(\mathbf{u}^{*}-\mathbf{u}_{h}^{*}, \mathbf{u}^{*}-\mathbf{u}_{h}^{*} \leqslant\right. & e c_{\varepsilon}(\mathbf{v})+e i_{\varepsilon}\left(\mathbf{v}_{h}\right)+a_{\varepsilon}\left(\mathbf{u}-\mathbf{u}_{h}, \mathbf{u}-\mathbf{v}_{h}\right) \\
& +b\left(\mathbf{v}_{h}-\mathbf{u}, p_{h}-p\right)+b\left(\mathbf{u}_{h}-\mathbf{u}, q_{h}-p\right)+c_{\varepsilon}\left(p-p_{h}, p-q_{h}\right) .
\end{aligned}
$$

Using Young inequality and the ellipticity of $a_{\varepsilon}(\cdot, \cdot)$ provide: $\forall \mathbf{v}^{*} \in \mathcal{K}, \forall \mathbf{v}_{h}^{*} \in \mathcal{K}_{h}$,

$$
\begin{aligned}
& \frac{1}{2} a_{\varepsilon}\left(\mathbf{u}-\mathbf{u}_{h}, \mathbf{u}-\mathbf{u}_{h}\right)+\frac{1}{2} c_{\varepsilon}\left(p-p_{h}, p-p_{h}\right) \\
& \leqslant \quad e c_{\varepsilon}(\mathbf{v})+e i_{\varepsilon}\left(\mathbf{v}_{h}\right)+\frac{1}{2}\left(1+\frac{\|b\|}{\delta \alpha}\right) a_{\varepsilon}\left(\mathbf{u}-\mathbf{v}_{h}, \mathbf{u}-\mathbf{v}_{h}\right)+\frac{1}{2} c_{\varepsilon}\left(p-q_{h}, p-q_{h}\right) \\
& \quad+\frac{\|b\|}{2 \alpha} \delta^{\prime} a_{\varepsilon}\left(\mathbf{u}-\mathbf{u}_{h}, \mathbf{u}-\mathbf{u}_{h}\right)+\frac{\|b\|}{2} \delta\left\|p-p_{h}\right\|_{M}^{2}+\frac{\|b\|}{2 \delta^{\prime}}\left\|p-q_{h}\right\|_{M}^{2} .
\end{aligned}
$$

On account of (3.9), we can write: $\forall \mathbf{v}^{*} \in \mathcal{K}, \forall \mathbf{v}_{h}^{*} \in \mathcal{K}_{h}$,

$$
\begin{aligned}
& a_{\varepsilon}\left(\mathbf{u}-\mathbf{u}_{h}, \mathbf{u}-\mathbf{u}_{h}\right)+c_{\varepsilon}\left(p-p_{h}, p-p_{h}\right) \\
& \leqslant 2\left(e c_{\varepsilon}(\mathbf{v})+e i_{\varepsilon}\left(\mathbf{v}_{h}\right)+\left(1+\frac{\|b\|}{\delta \alpha}\right) a_{\varepsilon}\left(\mathbf{u}-\mathbf{v}_{h}, \mathbf{u}-\mathbf{v}_{h}\right)+c_{\varepsilon}\left(p-q_{h}, p-q_{h}\right)\right. \\
& \quad+\frac{\|b\|}{\alpha}\left(\delta^{\prime}+2 \delta \eta^{2} a_{\varepsilon}\left(\mathbf{u}-\mathbf{u}_{h}, \mathbf{u}-\mathbf{u}_{h}\right)+\|b\|\left(\frac{1}{\delta^{\prime}}+2 \delta \eta^{2}\right)\left\|p-q_{h}\right\|_{M}^{2} .\right.
\end{aligned}
$$

Choosing $\delta$ and $\delta^{\prime}$ such that $\frac{\|b\|}{\alpha}\left(\delta^{\prime}+2 \delta \eta^{2}\right)=\frac{1}{2}$ and taking the infimum on $q_{h} \in M_{h}$, on $\mathbf{v} \in \mathbf{K}$ and on $\left\{\mathbf{v}_{h} \in \mathbf{K}_{h}, a_{1}\left(\mathbf{u}-\mathbf{v}_{h}, \mathbf{u}-\mathbf{v}_{h}\right)=0\right\}$ gives the estimate on $\left\|\mathbf{u}-\mathbf{u}_{h}\right\|_{\mathbf{X}}$. The estimate on $\left\|p-p_{h}\right\|_{M}$ is therefore a direct consequence of (3.9) and of the bound on $\left\|\mathbf{u}-\mathbf{u}_{h}\right\|_{\mathbf{X}}$.

Remark 3.2. Notice that the consistency error $\inf _{\mathbf{v} \in \mathbf{K}} e c_{\varepsilon}(\mathbf{v})$ is due to the nonconformity of the approximation. Otherwise if $\mathbf{K}_{h} \subset \mathbf{K}$ it suffices to choose $\mathbf{v}=\mathbf{u}_{h}$ to show that the infimum is $\leqslant 0$ and this error can be canceled in (3.8).

\section{Application to the mixed Signorini problem}

We are going to fit the mixed problem (2.6), (2.7) of the nearly incompressible elasticity to the abstract theory developed in the previous section and to describe and analyze its finite element approximation. 


\subsection{Well posedness of the continuous mixed Signorini problem}

Let us set $\mathbf{X}$ and $M$ to be respectively $H_{0}^{1}\left(\Omega, \Gamma_{\mathbf{u}}\right)^{2}$ and $L_{0}^{2}(\Omega)$. Then, define $a_{0}(\cdot, \cdot)$ and $a_{1}(\cdot, \cdot)$ to be the bilinear forms

$$
\begin{aligned}
& a_{0}(\mathbf{u}, \mathbf{v})=2 \mu\left(\boldsymbol{\varepsilon}(\mathbf{u}), \boldsymbol{\varepsilon}(\mathbf{v})_{L^{2}(\Omega)^{4}}, \quad \forall \mathbf{u}, \mathbf{v} \in \mathbf{X},\right. \\
& a_{1}(\mathbf{u}, \mathbf{v})=\frac{1}{|\Omega|}\left(\int_{\Gamma_{C}} \mathbf{u} \cdot \mathbf{n} \mathrm{d} \Gamma\right)\left(\int_{\Gamma_{C}} \mathbf{v} \cdot \mathbf{n} \mathrm{d} \Gamma\right), \quad \forall \mathbf{u}, \mathbf{v} \in \mathbf{X} .
\end{aligned}
$$

The current expression of $a_{1}(\cdot, \cdot)$ is obtained from the former one by Green's formula. For obvious commodity the bilinear forms $a_{\varepsilon}(\cdot, \cdot)$ with $\varepsilon=\frac{1}{\lambda}$ is rather denoted $a_{\lambda}(\cdot, \cdot)$ and $c_{\lambda}(\cdot, \cdot)$ (the same convention is adopted for the index) is defined to be: $\forall p, q \in M$,

$$
c_{\lambda}(p, q)=\frac{1}{\lambda} \int_{\Omega} p q \mathrm{~d} \mathbf{x} .
$$

Assumptions (i) and (ii) are readily checked (by Korn's inequality for (i) and with $\gamma=\tilde{\gamma}=1$ for (ii)). The construction of the space $\mathbf{W}$ from $\mathbf{K}$ shows that

$$
\mathbf{W}=\left\{\mathbf{v} \in \mathbf{X},(\mathbf{v} \cdot \mathbf{n})_{\mid \Gamma_{C}}=0,\right.
$$

which contains $\left(H_{0}^{1}(\Omega)\right)^{2}$. Then, hypothesis (iii) is straightforward from the standard inf-sup condition on $\left(H_{0}^{1}(\Omega)\right)^{2} \times L_{0}^{2}(\Omega)$ (see $[10,16]$ ) while hypothesis (iv) is directly obtained from the expression of $a_{1}(\cdot, \cdot)$. We are in position to apply Theorem 3.2 to establish existence, uniqueness and stability results for problem (2.6), (2.7).

Theorem 4.1. The mixed Signorini problem (2.6), (2.7) has only one solution $(\mathbf{u}, p) \in \mathbf{K} \times L_{0}^{2}(\Omega)$ that satisfies

$$
\|\mathbf{u}\|_{H^{1}(\Omega)^{2}}+\|p\|_{L^{2}(\Omega)} \leqslant C\|\mathbf{f}\|_{L^{2}(\Omega)^{2}}
$$

The constant $C$ is independent of $\lambda$.

Remark 4.1. For large values of $\lambda$, and in view of Remark 3.1 we have

$$
\left|\int_{\Omega} \operatorname{div} \mathbf{u} \mathrm{d} \mathbf{x}\right| \leqslant \frac{C}{\sqrt{\lambda}}\|\mathbf{f}\|_{L^{2}(\Omega)^{2}},
$$

while from the boundedness of $p$ we derive that

$$
\left\|\operatorname{div} \mathbf{u}-\frac{1}{|\Omega|}\left(\int_{\Omega} \operatorname{div} \mathbf{u} \mathrm{d} \mathbf{x}\right)\right\|_{L^{2}(\Omega)} \leqslant \frac{C}{\lambda}\|\mathbf{f}\|_{L^{2}(\Omega)^{2}} .
$$

This makes a small difference with the linear problem (when, e.g., $\Gamma_{C}=\emptyset$ ) where $\|\operatorname{div} \mathbf{u}\|_{L^{2}(\Omega)}$ decays like $\frac{1}{\lambda}$. 


\subsection{Taylor-Hood finite element approximation}

The finite element discretization of the mixed variational problem (2.6), (2.7) we choose to study is based on the Taylor-Hood finite elements constructed on triangular meshes. The analysis developed, hereafter, can be extended modulo some slight modifications to different type of finite elements such as, the MINI (or $\mathcal{P}_{1} /$ iso $\mathcal{P}_{1}$ ) finite element introduced by Bercovier and Pironneau, the Crouzeix-Raviart discontinuous $\mathcal{P}_{1} \times \mathcal{P}_{0}$ finite element or the stabilized Brezzi-Douglas-Marini (BDM) finite elements of (see [10]). The only point consists in the numerical modeling of the unilateral condition which should be enforced in an appropriate way so as to preserve the accuracy of the finite element used (see [4]).

Assume the shape of the domain $\Omega$ is polygonal so that it can be exactly covered by rectilinear finite elements. For any given discretization parameter $h>0$, let be given $\mathcal{T}_{h}$, a partition of $\Omega$ into triangles $\kappa$ with a maximum size $h$,

$$
\bar{\Omega}=\bigcup_{\kappa \in \mathcal{T}_{h}} \bar{\kappa}
$$

The analysis exposed here applies as well to the quadrangular finite elements. The set of the finite element nodes is $\Xi_{h}$. The family $\left(\mathcal{T}_{h}\right)_{h}$ is assumed to be $\mathcal{C}^{0}$-regular in the classical sense [11]. Moreover $\mathcal{T}_{h}$ is built in such a way that the boundar points $\left\{\mathbf{c}_{1}\right.$ and $\left.\mathbf{c}_{2}\right\}$ of $\Gamma_{C}$ are vertices of some triangles. For any $\kappa \in \mathcal{T}_{h}$, $\mathcal{P}_{r}(\kappa)$ stands for the set of polynomials of total degree $\leqslant r$. Then, we introduce the finite dimensional subspace $\mathbf{X}_{h}$ of $\mathbf{X}$ :

$$
\mathbf{X}_{h}=\mathbf{v}_{h} \in \mathcal{C}(\bar{\Omega})^{2}, \forall \kappa \in \mathcal{T}_{h}, \mathbf{v}_{h \mid \kappa} \in \mathcal{P}_{2}(\kappa)^{2}, \mathbf{v}_{h \mid \Gamma_{u}}=0
$$

The construction of the discrete convex cone requires the introduction of some more notations related to the contact zone. Due to the $\mathcal{C}^{0}$-regularity hypothesis, the boundary inherits a regular mesh $\mathcal{T}_{h}^{\partial \Omega}$, the elements of which are complete edges of the triangles $\kappa \in \mathcal{T}_{h}$. The trace of $\mathcal{T}_{h}^{\partial \Omega}$ on $\Gamma_{C}$ results in a mesh denoted by $\mathcal{T}_{h}^{C}$ and is characterized by the subdivision $\left(\mathbf{x}_{i}^{C}\right)_{0 \leqslant i \leqslant i^{*}}$ with $\mathbf{x}_{0}^{C}=\mathbf{c}_{1}$ and $\mathbf{x}_{i^{*}}^{C}=\mathbf{c}_{2}$ while $\left(t_{i}=\right] \mathbf{x}_{i}^{C}, \mathbf{x}_{i+1}^{C}[)_{0 \leqslant i \leqslant i^{*}-1}$ are its elements and the middle node of $t_{i}$ is denoted $\mathbf{x}_{i+1 / 2}^{C}$. To avoid high technicalities, in particular when fractional Sobolev norms are involved, so as to emphasize the specific features of the mixed formulation we assume that $\Gamma_{C}$ is a straight line. The generalization to a more complex geometry is readily checked at the cost of a longer mathematical analysis, which is beyond the scope of this work.

Following the choice made in [4], the numerical modeling of the Signorini condition consists in enforcing the nonpositivity of the values of the approximated normal displacement $\left(\mathbf{u}_{h} \cdot \mathbf{n}\right)$ at the vertices $\left(\mathbf{x}_{i}^{C}\right)_{0 \leqslant i \leqslant i^{*}}$ and on its integral value on the elements $\left(t_{i}\right)_{0 \leqslant i \leqslant i^{*}-1}$ instead of imposing the nonnegativity on the values of the approximated normal displacement $\left(\mathbf{u}_{h} \cdot \mathbf{n}\right)$ at the vertices $\left(\mathbf{x}_{i}^{C}\right)_{0 \leqslant i \leqslant i^{*}}$ and at the middle nodes $\left(\mathbf{x}_{i+1 / 2}\right)_{0 \leqslant i \leqslant i^{*}-1}$, which seems more natural. The reason why this approach is adopted will appear at the end of the current section. The finite dimensional closed convex cone of the admissible displacement fields is, then, defined to be

$$
\mathbf{K}_{h}=\left\{\mathbf{v}_{h} \in \mathbf{X}_{h},\left(\mathbf{v}_{h} \cdot \mathbf{n}\right)\left(\mathbf{x}_{i}^{C} \leqslant 0, \forall i\left(0 \leqslant i \leqslant i^{*} \int_{t_{i}}\left(\mathbf{v}_{h} \cdot \mathbf{n}\right) \mathrm{d} \Gamma \leqslant 0, \forall i\left(0 \leqslant i \leqslant i^{*}-1\right\} .\right.\right.\right.
$$


It is an easy matter to see that $\mathbf{K}_{h}$ is an external approximation of $\mathbf{K}$, i.e., $\mathbf{K}_{h} \not \subset \mathbf{K}$, thus the discretization is non-conforming. For technical needs we introduce the interpolation operator $\mathcal{J}_{h}$ which is specified by the following degrees of freedom

$$
\left(\mathbf{v}(\mathbf{x})_{\mathbf{x} \in \Xi_{h} \backslash \Gamma_{C}}, \quad\left(\mathbf { v } \left(\mathbf{x}_{i}^{C} \quad 0 \leqslant i \leqslant i^{*}, \quad\left(\int_{t_{i}} \mathbf{v} \mathrm{d} \Gamma\right)_{0 \leqslant i \leqslant i^{*}-1} .\right.\right.\right.
$$

Using the Bramble-Hilbert Theorem we can derive the following error estimate: For any $v(1<v \leqslant 3)$ there exists a constant $C>0$ such that: $\forall \mathbf{v} \in H^{v}(\Omega)^{2}$,

$$
\left\|\mathbf{v}-\mathcal{J}_{h} \mathbf{v}\right\|_{L^{2}(\Omega)^{2}}+h\left\|\mathbf{v}-\mathcal{J}_{h} \mathbf{v}\right\|_{H^{1}(\Omega)^{2}} \leqslant C h^{v}\|\mathbf{v}\|_{H^{v}(\Omega)^{2}} .
$$

A pleasant feature of this operator is that for any $\mathbf{v} \in \mathbf{K} \cap \mathcal{C}^{0}(\bar{\Omega})^{2}$ we have $\left(\mathcal{J}_{h} \mathbf{v}\right) \in \mathbf{K}_{h}$. To end with the description of the finite element framework, the discrete pressure is chosen to be in

$$
M_{h}=\left\{q_{h} \in \mathcal{C}(\bar{\Omega}), \forall \kappa \in \mathcal{T}_{h}, q_{h \mid \kappa} \in \mathcal{P}_{1}(\kappa), \int_{\Omega} q_{h} \mathrm{~d} \mathbf{x}=0\right\} .
$$

The discrete variational Signorini model is obtained as (3.6), (3.7), is set on the quadratic finite element closed cone $\mathbf{K}_{h}$ and the linear finite element space $M_{h}$ and reads as: find $\left(\mathbf{u}_{h}, p_{h}\right) \in \mathbf{K}_{h} \times M_{h}$ such that:

$$
\begin{aligned}
& a_{\lambda}\left(\mathbf{u}_{h}, \mathbf{v}_{h}-\mathbf{u}_{h}\right)+b\left(\mathbf{v}_{h}-\mathbf{u}_{h}, p_{h}\right) \geqslant \int_{\Omega} \mathbf{f} \cdot\left(\mathbf{v}_{h}-\mathbf{u}_{h}\right) \mathrm{d} \mathbf{x}, \quad \forall \mathbf{v}_{h} \in \mathbf{K}_{h}, \\
& b\left(\mathbf{u}_{h}, q_{h}\right)-c_{\lambda}\left(p_{h}, q_{h}\right)=0, \quad \forall q_{h} \in M_{h} .
\end{aligned}
$$

In order to check-up assumptions (iii) $)_{h}$ and (iv) $)_{h}$ we need to build the discrete space $\mathbf{W}_{h}: \mathbf{v}_{h} \in \mathbf{W}_{h}$ if and only if $\left(\mathbf{v}_{h} \cdot \mathbf{n}\right)\left(\mathbf{x}_{i}\right)=0\left(0 \leqslant i \leqslant i^{*}\right)$ and $\int_{t_{i}}\left(\mathbf{v}_{h} \cdot \mathbf{n}\right) \mathrm{d} \Gamma=0\left(0 \leqslant i \leqslant i^{*}-1\right)$. This yields that $\left(\mathbf{v}_{h} \cdot \mathbf{n}\right)_{\mid \Gamma_{C}}=0$; then $\mathbf{W}_{h} \subset \mathbf{W}$ and (iv) $)_{h}$ is fulfilled. The div-stability of $\left(\mathbf{W}_{h}, M_{h}\right)$ is issued from the classical result of the mixed Taylor-Hood finite elements, which says that a uniform inf-sup condition on $\mathbf{X}_{h} \cap\left(H_{0}^{1}(\Omega)\right)^{2}$ and $M_{h}$ is available (see [10]); this gives (iii) ${ }_{h}$. Theorem 3.2 can be applied and we then have

Proposition 4.2. The variational system (4.2), (4.3) is well posed and, then, has only one solution $\left(\mathbf{u}_{h}, p_{h}\right) \in \mathbf{K}_{h} \times M_{h}$ such that

$$
\left\|\mathbf{u}_{h}\right\|_{H^{1}(\Omega)^{2}}+\left\|p_{h}\right\|_{L^{2}(\Omega)} \leqslant C\|\mathbf{f}\|_{L^{2}(\Omega)^{2}} .
$$

The constant $C$ does not depend on $\lambda$.

\subsection{Error estimate}

The analysis of the accuracy of our mixed approximation shows that it is Poisson's locking-free. The convergence rate does not deteriorate for high values of $\lambda$ and is optimal with respect to the mesh-size $h$ (except in (4.7) where it suffers from the extra-term $|\log (h)|^{1 / 4}$ ) under reasonable regularity assumptions.

Theorem 4.3. Let $(\mathbf{u}, p) \in \mathbf{K} \times M$ be the solution of the mixed variational problem (2.6), (2.7). 
(i) Assume $\mathbf{u} \in H^{v}(\Omega)^{2}$ and $p \in H^{\nu-1}(\Omega)$ with $1<v \leqslant \frac{3}{2}$. Then, the discrete solution $\left(\mathbf{u}_{h}, p_{h}\right) \in \mathbf{K}_{h} \times$ $M_{h}$ satisfies

$$
\left\|\mathbf{u}-\mathbf{u}_{h}\right\|_{H^{1}(\Omega)^{2}}+\left\|p-p_{h}\right\|_{L^{2}(\Omega)} \leqslant C h^{\nu-1}\left(\|\mathbf{u}\|_{H^{\nu}(\Omega)^{2}}+\|p\|_{H^{\nu-1}(\Omega)}+\|\mathbf{f}\|_{L^{2}(\Omega)^{2}} .\right.
$$

(ii) Assume $\mathbf{u} \in H^{\nu}(\Omega)^{2}$ and $p \in H^{\nu-1}(\Omega)$ with $2<v \leqslant \frac{5}{2}$. Then, the discrete solution $\left(\mathbf{u}_{h}, p_{h}\right) \in \mathbf{K}_{h} \times$ $M_{h}$ satisfies

$$
\left\|\mathbf{u}-\mathbf{u}_{h}\right\|_{H^{1}(\Omega)^{2}}+\left\|p-p_{h}\right\|_{L^{2}(\Omega)} \leqslant C h^{\nu-1}\left(\|\mathbf{u}\|_{H^{v}(\Omega)^{2}}+\|p\|_{H^{\nu-1}(\Omega)} .\right.
$$

The constant $C$ in (4.4) and in (4.5) is independent of $\lambda$.

Theorem 4.4. Let $(\mathbf{u}, p) \in \mathbf{K} \times M$ be the solution of the mixed variational problem (2.6), (2.7), assume that the number of points in $\Gamma_{C}$, where the constraint changes from binding $(\mathbf{u} \cdot \mathbf{n}=0)$ to nonbinding $(\mathbf{u} \cdot \mathbf{n}<0)$, is finite.

(i) Assume $\mathbf{u} \in H^{v}(\Omega)^{2}$ and $p \in H^{\nu-1}(\Omega)$ with $\frac{3}{2}<v<2$. Then, the discrete solution $\left(\mathbf{u}_{h}, p_{h}\right) \in \mathbf{K}_{h} \times$ $M_{h}$ satisfies

$$
\left\|\mathbf{u}-\mathbf{u}_{h}\right\|_{H^{1}(\Omega)^{2}}+\left\|p-p_{h}\right\|_{L^{2}(\Omega)} \leqslant C h^{\nu-1}\left(\|\mathbf{u}\|_{H^{v}(\Omega)^{2}}+\|p\|_{H^{v-1}(\Omega)} .\right.
$$

(ii) Assume $\mathbf{u} \in H^{2}(\Omega)^{2}$ and $p \in H^{1}(\Omega)$. Then, the discrete solution $\left(\mathbf{u}_{h}, p_{h}\right) \in \mathbf{K}_{h} \times M_{h}$ satisfies

$$
\left\|\mathbf{u}-\mathbf{u}_{h}\right\|_{H^{1}(\Omega)^{2}}+\left\|p-p_{h}\right\|_{L^{2}(\Omega)} \leqslant C h|\log h|^{1 / 4}\left(\|\mathbf{u}\|_{H^{2}(\Omega)^{2}}+\|p\|_{H^{1}(\Omega)} .\right.
$$

The constant $C$ in (4.6) and in (4.7) is independent of $\lambda$.

Basically, the proof of both theorems is a combination of the sharp technical tools developed by Belhachmi and Ben Belgacem in [4] and the framework exposed in the previous section. Hereafter, we restrict ourselves to the detailed proof of the estimate (4.5). Those given in (4.4), (4.6) and (4.7) are proven following the same methodology.

First of all, for $\mathbf{u} \in H^{v}(\Omega)^{2}$ and $p \in H^{\nu-1}(\Omega)$ with $2<v \leqslant \frac{5}{2}$, a direct application to our problem of Theorem 3.3 and after transforming the terms $e i_{\lambda}\left(\mathbf{v}_{h}\right)$ and $e c_{\lambda}(\mathbf{v})$ by Green's formula we obtain

Proposition 4.5. The following error estimate holds

$$
\begin{aligned}
\| \mathbf{u}- & \mathbf{u}_{h}\left\|_{H^{1}(\Omega)^{2}}^{2}+\right\| p-p_{h} \|_{L^{2}(\Omega)}^{2} \\
\leqslant & C\left(\inf _{\substack{\mathbf{v}_{h} \in \mathbf{K}_{h} \\
\int_{C}\left(\mathbf{v}_{h} \cdot \mathbf{n}-\mathbf{u} \cdot \mathbf{n}\right) \mathrm{d} \Gamma=0}}\left[\left\|\mathbf{u}-\mathbf{v}_{h}\right\|_{H^{1}(\Omega)^{2}}^{2}+\int_{\Gamma_{C}} \sigma_{\mathbf{n}}^{\lambda}\left(\mathbf{v}_{h} \cdot \mathbf{n}-\mathbf{u} \cdot \mathbf{n}\right) \mathrm{d} \Gamma\right]\right. \\
& \left.+\inf _{\mathbf{v} \in \mathbf{K}} \int_{\Gamma_{C}} \sigma_{\mathbf{n}}^{\lambda}\left(\mathbf{v} \cdot \mathbf{n}-\mathbf{u}_{h} \cdot \mathbf{n}\right) \mathrm{d} \Gamma+\inf _{q_{h} \in M_{h}}\left\|p-q_{h}\right\|_{L^{2}(\Omega)}^{2}\right) .
\end{aligned}
$$

We are going to bound separately the best approximation error represented by the first infimum in (4.8) and the consistency error. 
Lemma 4.6. Let $(\mathbf{u}, p) \in \mathbf{K} \times M$ be the solution of the mixed variational Signorini problem (2.6), (2.7). Assume $\mathbf{u} \in H^{v}(\Omega)^{2}$ and $p \in H^{v-1}(\Omega)$ with $2<v \leqslant \frac{5}{2}$. Then

$$
\begin{aligned}
& \inf _{\substack{\mathbf{v}_{h} \in \mathbf{K}_{h} \\
\int_{\Gamma_{C}\left(\mathbf{v}_{h} \cdot \mathbf{n}-\mathbf{u} \cdot \mathbf{n}\right) \mathrm{d} \Gamma=0}}}\left[\left\|\mathbf{u}-\mathbf{v}_{h}\right\|_{H^{1}(\Omega)^{2}}^{2}+\int_{\Gamma_{C}} \sigma_{\mathbf{n}}^{\lambda}\left(\mathbf{v}_{h} \cdot \mathbf{n}-\mathbf{u} \cdot \mathbf{n}\right) \mathrm{d} \Gamma\right] \\
& \leqslant C h^{2(v-1)}\left(\|\mathbf{u}\|_{H^{v}(\Omega)^{2}}^{2}+\|p\|_{H^{v-1}(\Omega)}^{2} .\right.
\end{aligned}
$$

The constant $C$ is independent of $\lambda$.

Proof. Choosing $\mathbf{w}_{h}=\mathcal{J}_{h} \mathbf{u} \in \mathbf{K}_{h}$, then obviously, via the definition of $\mathcal{J}_{h}$, we have

$$
\int_{\Gamma_{C}}\left(\mathbf{w}_{h} \cdot \mathbf{n}-\mathbf{u} \cdot \mathbf{n}\right) \psi_{h} \mathrm{~d} \Gamma=0, \quad \forall \psi_{h} \in L^{2}\left(\Gamma_{C}\right), \psi_{h \mid t_{i}} \in \mathcal{P}_{0}\left(t_{i}\right) .
$$

In particular taking $\psi_{h}=1$, it holds that

$$
\int_{\Gamma_{C}}\left(\mathbf{w}_{h} \cdot \mathbf{n}-\mathbf{u} \cdot \mathbf{n}\right) \mathrm{d} \Gamma=0 .
$$

This makes $\mathbf{w}_{h}$ admissible to bound the infimum on the set $\left\{\mathbf{v}_{h} \in \mathbf{K}_{h}, \int_{\Gamma_{C}}\left(\mathbf{v}_{h} \cdot \mathbf{n}-\mathbf{u} \cdot \mathbf{n}\right) \mathrm{d} \Gamma=0\right\}$. Furthermore, using (4.1) we obtain

$$
\left\|\mathbf{u}-\mathbf{w}_{h}\right\|_{H^{1}(\Omega)^{2}}^{2} \leqslant C h^{2(\nu-1)}\|\mathbf{u}\|_{H^{\nu}(\Omega)^{2}}^{2} .
$$

Next, to estimate the integral term, observe right away that, since $\lambda$ is involved in $\sigma_{\mathbf{n}}^{\lambda}$ only through a constant term (see Remark 2.1) and because of (4.9), the dependency on $\lambda$ is canceled,

$$
\int_{\Gamma_{C}} \sigma_{\mathbf{n}}^{\lambda}\left(\mathbf{w}_{h} \cdot \mathbf{n}-\mathbf{u} \cdot \mathbf{n}\right) \mathrm{d} \Gamma=\int_{\Gamma_{C}} \sigma_{\mathbf{n}}\left(\mathbf{w}_{h} \cdot \mathbf{n}-\mathbf{u} \cdot \mathbf{n}\right) \mathrm{d} \Gamma .
$$

Let $\psi_{h}$ be fixed such that $\psi_{h \mid t_{i}}=\frac{1}{\left|t_{i}\right|} \int_{t_{i}} \sigma_{\mathbf{n}} \mathrm{d} \Gamma\left(0 \leqslant i \leqslant i^{*}\right)$, thanks to the construction of $\mathbf{w}_{h}$ we can write that

$$
\begin{aligned}
& \int_{\Gamma_{C}} \sigma_{\mathbf{n}}\left(\mathbf{w}_{h} \cdot \mathbf{n}-\mathbf{u} \cdot \mathbf{n}\right) \mathrm{d} \Gamma=\int_{\Gamma_{C}}\left(\sigma_{\mathbf{n}}-\psi_{h}\right)\left(\mathbf{w}_{h} \cdot \mathbf{n}-\mathbf{u} \cdot \mathbf{n}\right) \mathrm{d} \Gamma \\
& \quad \leqslant\left\|\sigma_{\mathbf{n}}-\psi_{h}\right\|_{L^{2}\left(\Gamma_{C}\right)}\left\|\mathbf{w}_{h} \cdot \mathbf{n}-\mathbf{u} \cdot \mathbf{n}\right\|_{L^{2}\left(\Gamma_{C}\right)} \leqslant C h^{\nu-3 / 2}\left\|\sigma_{\mathbf{n}}\right\|_{H^{\nu-3 / 2}\left(\Gamma_{C}\right)} h^{\nu-1 / 2}\|\mathbf{u} \cdot \mathbf{n}\|_{H^{\nu-1 / 2}\left(\Gamma_{C}\right)} .
\end{aligned}
$$

Then, the proof is completed by the trace theorem.

We cope, now, with the consistency error so as to derive an intermediary bound of it. By a bootstrapping, this bound allows to state a final estimate.

Lemma 4.7. Let $(\mathbf{u}, p) \in \mathbf{K} \times M$ be the solution of the mixed variational Signorini problem (2.6), (2.7). Assume $\mathbf{u} \in H^{v}(\Omega)^{2}$ and $p \in H^{\nu-1}(\Omega)$ with $2<v \leqslant \frac{5}{2}$. Then

$$
\inf _{\mathbf{v} \in \mathbf{K}} \int_{\Gamma_{C}} \sigma_{\mathbf{n}}^{\lambda}\left(\mathbf{v} \cdot \mathbf{n}-\mathbf{u}_{h} \cdot \mathbf{n}\right) \mathrm{d} \Gamma \leqslant C h^{\nu-1}\left(\left\|\mathbf{u}-\mathbf{u}_{h}\right\|_{\mathbf{X}}+h^{\nu-1}\|\mathbf{u}\|_{H^{\nu}(\Omega)^{2}}\right)\left(\|\mathbf{u}\|_{H^{\nu}(\Omega)^{2}}+\|p\|_{H^{\nu-1}(\Omega)} .\right.
$$

The constant $C$ is independent of $\lambda$. 
Proof. Taking $\mathbf{v}=\mathbf{u} \in \mathbf{K}$ and choosing $\psi_{h}^{\lambda}$ such that $\psi_{h \mid t_{i}}^{\lambda}=\frac{1}{\left|t_{i}\right|} \int_{t_{i}} \sigma_{\mathbf{n}}^{\lambda} \mathrm{d} \Gamma\left(0 \leqslant i \leqslant i^{*}\right)$, we have

$$
\begin{aligned}
& \int_{\Gamma_{C}} \sigma_{\mathbf{n}}^{\lambda}\left(\mathbf{u} \cdot \mathbf{n}-\mathbf{u}_{h} \cdot \mathbf{n}\right) \mathrm{d} \Gamma \\
& \quad=\int_{\Gamma_{C}}\left(\sigma_{\mathbf{n}}^{\lambda}-\psi_{h}^{\lambda}\left(\mathbf{u} \cdot \mathbf{n}-\mathbf{u}_{h} \cdot \mathbf{n}\right) \mathrm{d} \Gamma+\int_{\Gamma_{C}} \psi_{h}^{\lambda}\left(\mathbf{u} \cdot \mathbf{n}-\mathbf{u}_{h} \cdot \mathbf{n}\right) \mathrm{d} \Gamma .\right.
\end{aligned}
$$

To bound the first part observe that

$$
\psi_{h}^{\lambda}=\psi_{h}+\frac{\lambda}{|\Omega|} \int_{\Omega} \operatorname{div} \mathbf{u} \mathrm{d} \mathbf{x}
$$

with $\psi_{h \mid t_{i}}=\frac{1}{\left|t_{i}\right|} \int_{t_{i}} \sigma_{\mathbf{n}} \mathrm{d} \Gamma\left(0 \leqslant i \leqslant i^{*}\right)$. Then, we deduce that $\sigma_{\mathbf{n}}^{\lambda}-\psi_{h}^{\lambda}=\sigma_{\mathbf{n}}-\psi_{h}$ and

$$
\begin{aligned}
& \int_{\Gamma_{C}}\left(\sigma_{\mathbf{n}}^{\lambda}-\psi_{h}^{\lambda}\left(\mathbf{u} \cdot \mathbf{n}-\mathbf{u}_{h} \cdot \mathbf{n}\right) \mathrm{d} \Gamma \leqslant\left\|\sigma_{\mathbf{n}}-\psi_{h}\right\|_{H_{00}^{1 / 2}\left(\Gamma_{C}\right)^{\prime}}\left\|\mathbf{u} \cdot \mathbf{n}-\mathbf{u}_{h} \cdot \mathbf{n}\right\|_{H_{00}^{1 / 2}\left(\Gamma_{C}\right)}\right. \\
& \quad \leqslant C h^{\nu-1}\left\|\sigma_{\mathbf{n}}\right\|_{H^{\nu-3 / 2}\left(\Gamma_{C}\right)}\left\|\mathbf{u} \cdot \mathbf{n}-\mathbf{u}_{h} \cdot \mathbf{n}\right\|_{H_{00}^{1 / 2}\left(\Gamma_{C}\right)} \leqslant C h^{\nu-1}\left(\|\mathbf{u}\|_{H^{\nu}(\Omega)}+\|p\|_{H^{\nu-1}(\Omega)}\left\|\mathbf{u}-\mathbf{u}_{h}\right\| \mathbf{X} .\right.
\end{aligned}
$$

The estimate on $\left(\sigma_{\mathbf{n}}-\psi_{h}\right)$ can be found in [2]. To handle the second term of (4.10) notice that

$$
\int_{\Gamma_{C}} \psi_{h}^{\lambda}\left(\mathbf{u}_{h} \cdot \mathbf{n}\right) \mathrm{d} \Gamma=\sum_{i=0}^{i^{*}-1} \psi_{h \mid t_{i}}^{\lambda} \int_{t_{i}} \mathbf{u}_{h} \cdot \mathbf{n} \mathrm{d} \Gamma \geqslant 0 .
$$

This yields that

$$
\int_{\Gamma_{C}} \psi_{h}^{\lambda}\left(\mathbf{u} \cdot \mathbf{n}-\mathbf{u}_{h} \cdot \mathbf{n}\right) \mathrm{d} \Gamma \leqslant \int_{\Gamma_{C}} \psi_{h}^{\lambda}(\mathbf{u} \cdot \mathbf{n}) \mathrm{d} \Gamma .
$$

Define $\chi_{h} \in L^{2}\left(\Gamma_{C}\right)$ such that $\chi_{h \mid t_{i}}=\frac{1}{\left|t_{i}\right|} \int_{t_{i}}(\mathbf{u} \cdot \mathbf{n}) \mathrm{d} \Gamma\left(0 \leqslant i \leqslant i^{*}\right)$, in view of the saturation $\sigma_{\mathbf{n}}^{\lambda}(\mathbf{u} \cdot \mathbf{n})=0$ on $\Gamma_{C}$, we can write that

$$
\int_{\Gamma_{C}} \psi_{h}^{\lambda}(\mathbf{u} \cdot \mathbf{n}) \mathrm{d} \Gamma=\int_{\Gamma_{C}}\left(\psi_{h}^{\lambda}-\sigma_{\mathbf{n}}^{\lambda}\left(\mathbf{u} \cdot \mathbf{n}-\chi_{h}\right) \mathrm{d} \Gamma=\sum_{i=0}^{i^{*}-1} \int_{t_{i}}\left(\psi_{h}^{\lambda}-\sigma_{\mathbf{n}}^{\lambda}\left(\mathbf{u} \cdot \mathbf{n}-\chi_{h}\right) \mathrm{d} \Gamma .\right.\right.
$$

The sum can be restricted to the set $I$ of indices $i$ for which $\mathbf{u} \cdot \mathbf{n}$ vanishes at least once in $t_{i}$. Indeed, if $(\mathbf{u} \cdot \mathbf{n})_{\mid t_{i}}<0$ then $\boldsymbol{\sigma}_{\mathbf{n} \mid t_{i}}^{\lambda}=0$ this yields $\psi_{h \mid t_{i}}^{\lambda}=0$, and therefore the integral on $t_{i}$ vanishes. Then

$$
\begin{aligned}
\int_{\Gamma_{C}} \psi_{h}^{\lambda}(\mathbf{u} \cdot \mathbf{n}) \mathrm{d} \Gamma & =\sum_{i \in I} \int_{t_{i}}\left(\psi_{h}^{\lambda}-\sigma_{\mathbf{n}}^{\lambda}\left(\mathbf{u} \cdot \mathbf{n}-\chi_{h}\right) \mathrm{d} \Gamma=\sum_{i \in I} \int_{t_{i}}\left(\psi_{h}-\sigma_{\mathbf{n}}\right)\left(\mathbf{u} \cdot \mathbf{n}-\chi_{h}\right) \mathrm{d} \Gamma\right. \\
& \leqslant \sum_{i \in I}\left\|\psi_{h}-\sigma_{\mathbf{n}}\right\|_{L^{2}\left(t_{i}\right)}\left\|\mathbf{u} \cdot \mathbf{n}-\chi_{h}\right\|_{L^{2}\left(t_{i}\right)} \leqslant C \sum_{i \in I} h_{i}^{\nu-3 / 2}\left\|\boldsymbol{\sigma}_{n}\right\|_{H^{\nu-3 / 2}\left(t_{i}\right)} h_{i}|\mathbf{u} \cdot \mathbf{n}|_{H^{1}\left(t_{i}\right)} .
\end{aligned}
$$

The evaluation of the semi-norm $|\mathbf{u} \cdot \mathbf{n}|_{H^{1}\left(t_{i}\right)}$ is carried out as in [4, Lemma 4.11]. Since $(\mathbf{u} \cdot \mathbf{n})_{\mid t_{i}} \in \mathcal{C}^{1}\left(t_{i}\right)$ with $(\mathbf{u} \cdot \mathbf{n})_{\mid t_{i}} \leqslant 0$ and vanishes at least once in $t_{i}$ then

$$
|\mathbf{u} \cdot \mathbf{n}|_{H^{1}\left(t_{i}\right)} \leqslant C h_{i}^{\nu-3 / 2}\|\mathbf{u} \cdot \mathbf{n}\|_{H^{\nu-1 / 2}\left(t_{i}\right)},
$$


so that

$$
\begin{aligned}
\int_{\Gamma_{C}} \psi_{h}^{\lambda}(\mathbf{u} \cdot \mathbf{n}) \mathrm{d} \Gamma & \leqslant \sum_{i \in I} C h_{i}^{\nu-3 / 2}\left\|\sigma_{\mathbf{n}}\right\|_{H^{\nu-3 / 2}\left(t_{i}\right)} h_{i}^{\nu-1 / 2}\|\mathbf{u} \cdot \mathbf{n}\|_{H^{\nu-1 / 2}\left(t_{i}\right)} \\
& \leqslant C h^{2(\nu-1)}\left(\sum_{i \in I}\left\|\sigma_{\mathbf{n}}\right\|_{H^{\nu-3 / 2}\left(t_{i}\right)}^{2}\right)^{1 / 2}\left(\sum_{i \in I}\|\mathbf{u} \cdot \mathbf{n}\|_{H^{\nu-1 / 2}\left(t_{i}\right)}^{2}\right)^{1 / 2} \\
& \leqslant C h^{2(\nu-1)}\left\|\sigma_{\mathbf{n}}\right\|_{H^{\nu-3 / 2}\left(\Gamma_{C}\right)}\|\mathbf{u} \cdot \mathbf{n}\|_{H^{\nu-1 / 2}\left(\Gamma_{C}\right)} \\
& \leqslant C h^{2(\nu-1)}\left(\|\mathbf{u}\|_{H^{\nu}(\Omega)^{2}}+\|p\|_{H^{\nu-1}(\Omega)}\|\mathbf{u}\|_{H^{\nu}(\Omega)} .\right.
\end{aligned}
$$

The proof is complete.

Proof of (4.3) of Theorem 4.3. Putting together Lemmas 4.6 and 4.7 we obtain that

$$
\begin{aligned}
& \left\|\mathbf{u}-\mathbf{u}_{h}\right\|_{H^{1}(\Omega)^{2}}^{2}+\left\|p-p_{h}\right\|_{L^{2}(\Omega)}^{2} \\
& \quad \leqslant C\left(h^{2(\nu-1)}\|p\|_{H^{\nu-1}(\Omega)}^{2}+h^{\nu-1}\left(\left\|\mathbf{u}-\mathbf{u}_{h}\right\|_{H^{1}(\Omega)^{2}}+h^{\nu-1}\|\mathbf{u}\|_{H^{\nu}(\Omega)^{2}}\right)\left(\|\mathbf{u}\|_{H^{\nu}(\Omega)^{2}}+\|p\|_{H^{\nu-1}(\Omega)}\right.\right.
\end{aligned}
$$

from which (4.5) of Theorem 4.3 is issued.

Remark 4.2. In [4], a second numerical model—called the pointwise model—for the Signorini conditions that looks more natural is studied. The corresponding discrete closed convex cone is defined as follows

$$
\mathbf{H}_{h}=\mathbf{v}_{h} \in \mathbf{X}_{h},\left(\mathbf{v}_{h} \cdot \mathbf{n}\right)\left(\mathbf{x}_{i}^{C} \leqslant 0, \forall i\left(0 \leqslant i \leqslant i^{*}\left(\mathbf{v}_{h} \cdot \mathbf{n}\right)\left(\mathbf{x}_{i+1 / 2}^{C} \leqslant 0, \forall i\left(0 \leqslant i \leqslant i^{*}-1 .\right.\right.\right.\right.
$$

In the compressible elasticity, when a primal formulation is used with the displacement $\mathbf{u}$ as the only unknown, it is shown that this model performs as well as the one adopted in this paper-the integral model. In the mixed nearly incompressible elasticity things are changed. Indeed, when only Signorini and Dirichlet boundary conditions are enforced on the boundary $\Gamma$ the pointwise model fails to provide locking-free results. In particular the result of Lemma 4.6 does not hold any longer. The reason is that if we choose $\mathbf{v}_{h}=\mathcal{I}_{h} \mathbf{u} \in \mathbf{H}_{h}$ in the proof of that lemma, $\mathcal{I}_{h}$ being the classical Lagrange interpolation operator, the identity $\int_{\Gamma_{C}}\left(\mathbf{v}_{h} \cdot \mathbf{n}-\mathbf{u} \cdot \mathbf{n}\right) \mathrm{d} \Gamma=0$ is not valid anymore. In addition the bound of the integral term $e i_{\lambda}\left(\mathbf{v}_{h}\right)$ is polluted by $\lambda$.

However, most often a part of the boundary is subjected to Neumann condition, then there is no need to split $(\lambda \operatorname{div} \mathbf{u})$ as in $(2.5)$ and setting $p=(\lambda \operatorname{div} \mathbf{u}) \in L^{2}(\Omega)$ becomes possible; the mixed variational model is modified and is easier to analyze (see [26] for details).

Remark 4.3. The extension of our study to the MINI finite elements $\mathcal{P}_{1} /$ iso $\mathcal{P}_{1}$ or to the stabilized BDM finite elements is readily checked. The only point is to define properly the discrete Signorini conditions. The discrete spaces $\mathbf{K}_{h}$ and $M_{h}$ are constructed on two overlapping meshes. The pressure triangles are denoted $\kappa_{p}$ and $p_{h \mid \kappa_{p}} \in \mathcal{P}_{1}$. Each $\kappa_{p}$ is broken into four smaller triangles $\kappa_{\mathbf{u}}$ in the way shown in Fig. 2, and $\mathbf{u}_{h \mid \kappa_{\mathbf{u}}} \in \mathcal{P}_{1}$. 


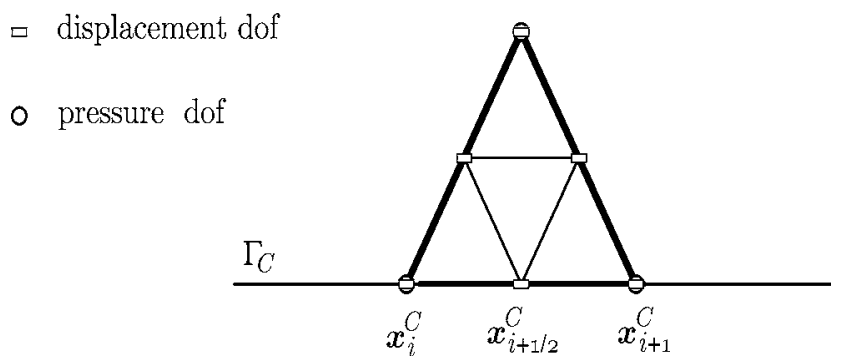

Fig. 2.

The set of the pressure points located on $\Gamma_{C}$ are $\left(\mathbf{x}_{i}^{C}\right)_{0 \leqslant i \leqslant i^{*}}$ while those determining the degrees of freedom of $\mathbf{u}_{h}$ are $\left(\mathbf{x}_{i}^{C}\right)_{0 \leqslant i \leqslant i^{*}}$ and $\left(\mathbf{x}_{i+1 / 2}^{C}\right)_{0 \leqslant i \leqslant i^{*}-1}$. Then, the numerical modeling of the Signorini conditions reads as

$$
\left(\mathbf{u}_{h} \cdot \mathbf{n}\right)\left(\mathbf{x}_{i}^{C} \leqslant 0 \quad\left(0 \leqslant i \leqslant i^{*} \quad \int_{\mathbf{x}_{i}^{C}}^{\mathbf{x}_{i+1}^{C}}\left(\mathbf{u}_{h} \cdot \mathbf{n}\right) \mathrm{d} \Gamma \leqslant 0 \quad\left(0 \leqslant i \leqslant i^{*}-1 .\right.\right.\right.
$$

In this case the analysis may be carried out in the same way as done in this work and the results announced in part (i) of Theorem 4.3 and in Theorem 4.4 still hold.

\section{Implementation and numerical discussion}

Before discussing some examples to illustrate the conditions where the numerical locking occurs for the Signorini problem and how to overcome it via the mixed Taylor-Hood finite element approximation, we provide some hints on the implementation and we describe briefly the algorithm used to solve the discrete problem (4.2), (4.3).

Let $\underline{\mathbf{u}}_{h} \in \mathbb{R}^{n}$ denote the discrete displacement vector whose components are $\left(\mathbf{u}_{h}(\mathbf{x}), \mathbf{x} \in \Xi_{h} \backslash \Gamma_{D}\right)$, then $n=2 \operatorname{card}\left(\Xi_{h} \backslash \Gamma_{D}\right)$, card is the cardinality, $\underline{\mathbf{p}}_{h} \in \mathbb{R}^{m}$ stands for the vector of $\left(p_{h}(\mathbf{x}), \mathbf{x} \in \Xi_{h}\right)$, with $m=$ card $\Xi_{h}$, where we give up, at least for a while, the zero mean value constraint. The Signorini conditions on the contact border specifying the admissible displacements can be expressed by the means of a rectangular matrix $M_{h}^{C} ; \mathbf{v}_{h} \in \mathbf{K}_{h}$ if its vector representation $\underline{\mathbf{v}}_{h}$ satisfies the inequality $M_{h}^{C} \underline{\mathbf{v}}_{h} \leqslant 0$, in the sense that each component of it is nonpositive. Using these stencils, the algebraic equivalent of (4.3), (4.4) reads as: find $\underline{\mathbf{u}}_{h} \in \mathbb{R}^{n}, \underline{\mathbf{p}}_{h} \in \mathbb{R}^{m}$, with $M_{h}^{C} \underline{\mathbf{u}}_{h} \leqslant 0$ and

$$
\begin{aligned}
& \left(A_{\lambda, h} \underline{\mathbf{u}}_{h}, \underline{\mathbf{v}}_{h}-\underline{\mathbf{u}}_{h}\right)_{\mathbb{R}^{n}}+\left(B_{h}^{\mathrm{T}} \underline{\mathbf{p}}_{h}, \underline{\mathbf{v}}_{h}-\underline{\mathbf{u}}_{h} \mathbb{R}^{n} \geqslant\left(\underline{\mathbf{l}}_{h}, \underline{\mathbf{v}}_{h}-\underline{\mathbf{u}}_{h}\right)_{\mathbb{R}^{n}}, \quad \forall \underline{\mathbf{v}}_{h} \in \mathbb{R}^{n}, M_{h}^{C} \underline{\mathbf{v}}_{h} \leqslant 0,\right. \\
& B_{h} \underline{\mathbf{u}}_{h}-\frac{1}{\lambda} M_{h} \underline{\mathbf{p}}_{h}=0 .
\end{aligned}
$$

In (5.1), $(\cdot, \cdot)_{\mathbb{R}^{n}}$ is the inner product in $\mathbb{R}^{n}, A_{\lambda, h}=A_{0}+\lambda A_{1}$ the matrix of the bilinear form $a_{\lambda}(\cdot, \cdot)$ which is symmetric and positive definite, the matrix $B_{h}$ is associated with $b(\cdot, \cdot),{ }^{\mathrm{T}}$ is the transposition symbol, $M_{h}$ is the mass matrix for the hydrostatic pressure and the vector $\underline{\mathbf{l}}_{h} \in \mathbb{R}^{n}$ is the representation 
of the exterior load involved in the linear form $\ell$. The system (5.1), (5.2) can be viewed as the optimality conditions of the min-max problem

$$
\underline{\mathcal{L}}\left(\underline{\mathbf{u}}_{h}, \underline{\mathbf{p}}_{h}\right)=\min _{M_{h}^{C} \underline{\mathbf{v}}_{h} \leqslant 0} \max _{\underline{\mathbf{q}}_{h}} \underline{\mathcal{L}}\left(\underline{\mathbf{v}}_{h}, \underline{\mathbf{q}}_{h}\right),
$$

$\left(\underline{\mathbf{u}}_{h}, \underline{\mathbf{p}}_{h}\right)$ being the saddle point of the Lagrangian functional given by

$$
\underline{\mathcal{L}}\left(\underline{\mathbf{v}}_{h}, \underline{\mathbf{q}}_{h}\right)=\frac{1}{2}\left(A_{\lambda, h} \underline{\mathbf{v}}_{h}, \underline{\mathbf{v}}_{h}\right)_{\mathbb{R}^{n}}+\left(\mathbf{q}_{h}, B_{h} \underline{\mathbf{v}}_{h}\right)_{\mathbb{R}^{n}}-\left(\underline{\mathbf{l}}_{h}, \underline{\mathbf{v}}_{h}\right)_{\mathbb{R}^{n}}
$$

The solver adopted in our $\mathrm{C}_{++}$finite element code GETFEM $_{++}$(see [25]) is based on the Uzawa algorithm (see [14]). We build up a sequence $\left(\underline{\mathbf{u}}_{h}^{r}, \underline{\mathbf{p}}_{h}^{r}\right)_{r}$ in a recurrent way, $\underline{\mathbf{p}}_{h}^{r}$ being known, we compute $\underline{\mathbf{u}}_{h}^{r+1}$ as the solution of the convex optimization problem

$$
\underline{\mathcal{L}}\left(\underline{\mathbf{u}}_{h}^{r+1}, \underline{\mathbf{p}}_{h}^{r}=\min _{M_{h}^{C} \underline{\mathbf{v}}_{h} \leqslant 0} \underline{\mathcal{L}}\left(\underline{\mathbf{v}}_{h}, \underline{\mathbf{p}}_{h}^{r} .\right.\right.
$$

Then, the pressure is updated as follows

$$
M_{h} \underline{\mathbf{p}}_{h}^{r+1}=M_{h} \underline{\mathbf{p}}_{h}^{r}+\rho^{r} B_{h} \underline{\mathbf{u}}^{r+1},
$$

for some coefficient $\rho^{r}$ appropriately chosen to ensure the convergence of the iterative process. The minimization problem (5.3) is handled using the Polak-Ribière Conjugate Gradient method with a linesearch (see [24,8]), the details of this procedure so as of some others employed to solve the approximated mixed problem will be exposed in [7]. Observe that even though $A_{\lambda, h}$ depends on the parameter $\lambda$, the convergence speed of the internal CG-solver of the minimization problem (5.3) is not affected by high values of that parameter. Indeed, only a single eigenvalue of the matrix $A_{\lambda, h}$ grows like $\lambda$ and it is well known that the CG method cancels the component of the residual in the corresponding eigen-direction in one step, most often in the first internal iterations for high eigenvalues. Recall that when the elastic body is submitted to a Neumann condition along a part of its boundary there is no need that the hydrostatic pressure be null-averaged. In this case, the term $\lambda\left(\int_{\Gamma_{C}} \mathbf{u} \cdot \mathbf{n}\right)\left(\int_{\Gamma_{C}} \mathbf{v} \cdot \mathbf{n}\right)$ depending on $\lambda$ is taken into account by aggragating it to the pressure $p$, and instead of $A_{\lambda, h}$ we have a matrix independent of $\lambda$.

The numerical tests we present are realized on a square-shaped elastic body $\Omega=(0,1)^{2}$ which is originally in rest on the ground considered as a rigid foundation. The solid is slightly and uniformly lifted from its above edge, it may be modeled by a Dirichlet boundary condition $\mathbf{u}=\mathbf{u}_{D}=(0, \alpha)$ imposed on $\Gamma_{D}=\{1\} \times[0,1]$. Under the effect of its own weight the solid undergoes an elastic deformation and a part of its bottom edge $\Gamma_{C}=\{0\} \times[0,1]$ may leave the ground, therefore the Signorini boundary conditions are recommended on $\Gamma_{C}$. On the vertical edges the body is free of any external solicitation and is then subjected to a homogeneous Neumann condition. Fig. 3 shows, with an exaggerated scale, the shape of the solid before (still a squared shape) and after deformation (the body is made thinner on its above half while the extreme portions take off the ground).

For different materials, having different properties of incompressibility (the associated Poisson coefficients are different) and for the standard $\mathcal{P}_{1}, \mathcal{P}_{2}$ and for the mixed $\mathcal{P}_{2} \times \mathcal{P}_{1}$ Taylor-Hood approximations we depict, in logarithmic scales, the maximum of the error (at the mesh points) on the displacement field with respect to the mesh size. In each case, the error is evaluated by comparing the computed solution on the current mesh to a reference displacement calculated by the Taylor-Hood finite element approximation using a sufficient fine mesh (each edge is subdivided into 256 elements). Actually, the computation resolution for the reference solution is fixed after having measured the gap between the Taylor-Hood 


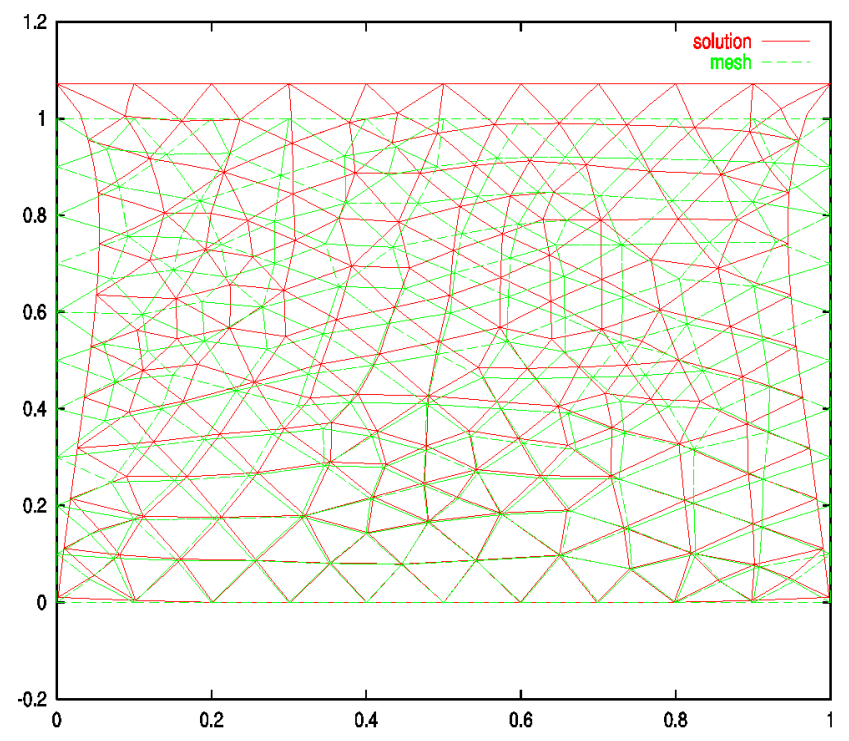

Fig. 3. Shape of the elastic solid before (dashed lines) and after deformation (continuous lines).

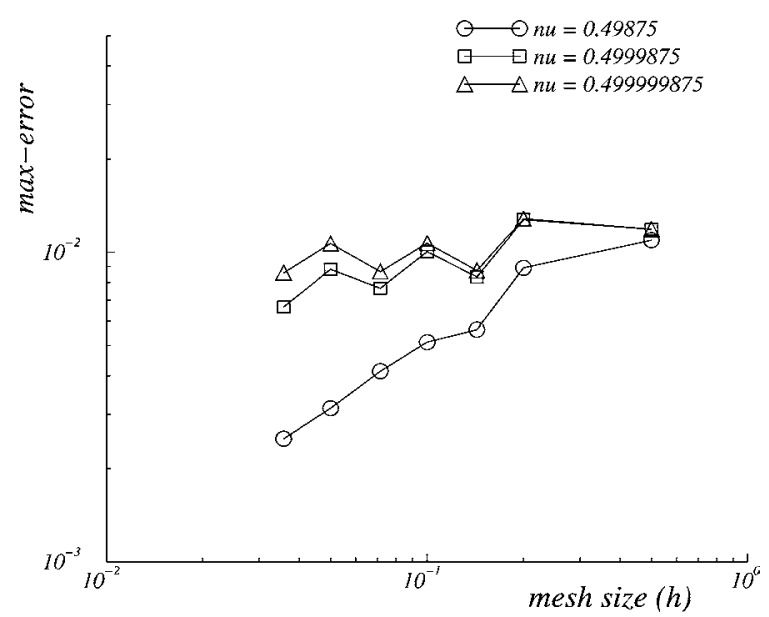

Fig. 4. Numerical locking of the linear finite element displacement.

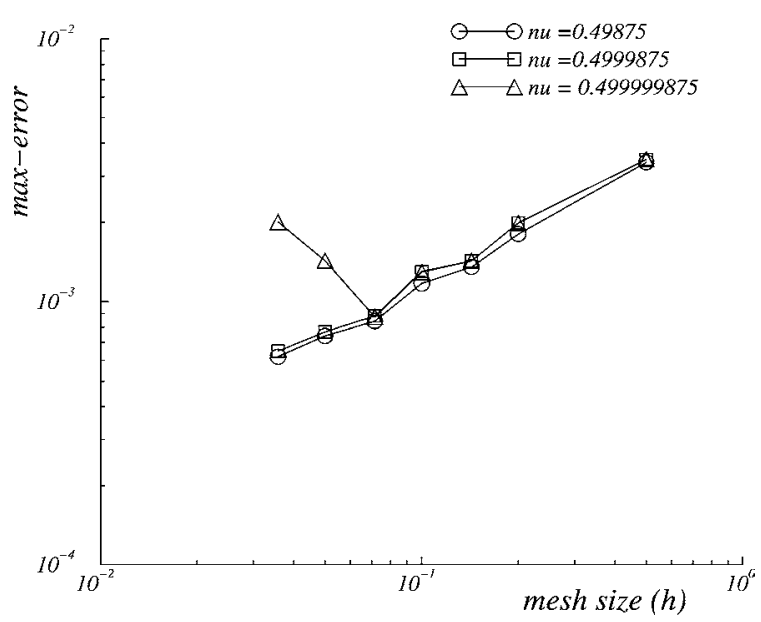

Fig. 5. Accuracy of the quadratic finite element displacement. The deterioration for $v=0.499999875$ is caused by the ill-conditioning of the system.

finite element displacement with that obtained by a $\mathcal{P}_{4}$ standard finite element displacement where the Signorini condition is enforced point-wisely on $\Gamma_{C}$. The difference between both solutions is sufficiently small which makes us confident in the quality of the computed displacement on that fine mesh; we adopt it as a reference solution since it is widely known that, for the more classical Dirichlet/Neumann conditions, the high order finite elements is locking-free in nearly incompressible elasticity. Notice that for both $\mathcal{P}_{1}$ - and $\mathcal{P}_{2}$-approximations the displacement is calculated as the finite element solution of the standard Signorini variational inequality by the Polak-Ribière CG procedure. 


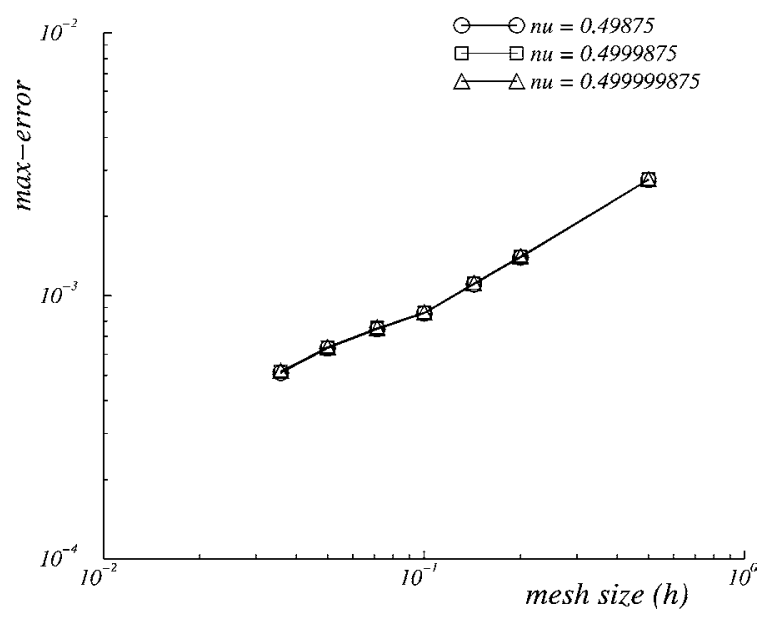

Fig. 6. Monotonic decaying of the error of the Taylor-Hood finite element displacement. No locking and no alteration caused by the conditioning of the system are observed.

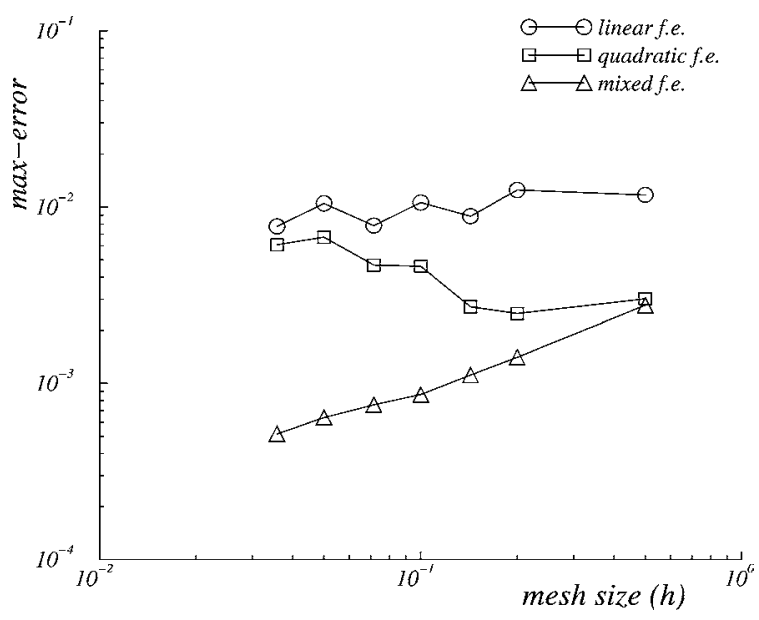

Fig. 7. Convergence curves for the standard finite element and the Taylor-Hood element solutions.

The effect of the Poisson numerical locking when $v$ comes close to $\frac{1}{2}$ (equivalently when $\lambda$ goes to infinity) can be observed in Fig. 4. For $v=0.4999875$ and $v=0.499999875$ there is no longer a decrease of the error given by the linear approximation as we remark that the corresponding curve is almost flat.

The quadratic discretization, leads to a better accuracy, which is illustrated in Fig. 5, but we remark that for $v=0.499999875$ a significant deterioration appears when the number of the degrees of freedom increases. To our opinion, the reason of such an undesirable behavior is that the CG fails to calculate a satisfactory solution of the Signorini variational inequality because the condition number of the stiffness matrix is drastically increased.

In the contrary, Fig. 6 shows that for the mixed Taylor-Hood finite element solution we did not observe any slow down of the convergence rate of the internal CG iterative solver nor of the Uzawa algorithm. The mixed finite element error on the reference displacement decays monotonically, which expresses that it is locking-free.

Finally, we plot in the same frame (of Fig. 7) the convergence curves when the Poisson coefficient $v=0.49999999875$ in order to stress the trends observed for the previous experiences and to have a better insight on the efficiency of the mixed approach. The linear finite element displacement suffers from numerical locking, the condition number of the quadratic finite element system seems even worse and we are not able to solve it accurately, the bad impact on the computed displacement is clearly remarked. The mixed approximation still give satisfactory results.

\section{Concluding remarks and future work}

An efficient device to take into account the (near) incompressibility for the Signorini system in elasticity is to use the hydrostatic pressure as an independent variable, in addition to the displacement field. The resulting mixed formulation involves a variational inequality expressing the motion equation together with a variational equation stating the connection between the pressure and the displacement. The study 
of this system requires a substantial adaptation of the saddle point theory to the case of inequalities. This aim is brought into a successful conclusion in this work. Indeed, the abstract framework presented fits to the Signorini problem in nearly incompressible elasticity, where the well posedness is obtained and the Taylor-Hood discretization provides the expected optimal convergence rates. Some numerical experiences discussed in the previous section are in accordance with the theoretical predictions.

This paper may stimulate some theoretical work in several directions for the unilateral contact. For instance, an interesting challenge is the theoretical analysis of the matching of meshes for the numerical simulation of the displacement of two elastic bodies subjected to a unilateral contact along a common zone, when one of them is at least constituted of (nearly) incompressible material (see [6,21,5]). This represents one of the future perspectives of our team.

\section{References}

[1] R.A. Adams, Sobolev Spaces, Academic Press, New York, 1975.

[2] I. Babuška, A.K. Aziz, Survey lectures on the mathematical foundations of the finite element method. With the collaboration of G. Fix and R.B. Kellogg. The mathematical foundations of the finite element method with applications to partial differential equations, in: Proc. Sympos., Univ. Maryland, Baltimore, MD, 1972, Academic Press, New York, 1972, pp. 1359.

[3] I. Babuška, M. Suri, Locking effects in the finite element approximation of elasticity problem, Numer. Math. 62 (1992) 439-463.

[4] Z. Belhachmi, F. Ben Belgacem, Quadratic finite element approximation of the Signorini problem, Math. Comp. 72 (2003) 83-104.

[5] F. Ben Belgacem, Numerical simulation of some variational inequalities arisen from unilateral contact problems by the finite element method, SIAM J. Numer. Anal. 37 (2000) 1198-1216.

[6] F. Ben Belgacem, P. Hild, P. Laborde, Extension of the mortar finite element to a variational inequality modeling unilateral contact, Math. Models Methods Appl. Sci. 9 (1999) 287-303.

[7] F. Ben Belgacem, Y. Renard, On the numerical solutions of the Signorini problem by various finite element methods, in preparation.

[8] J.-F. Bonnans, J.-C. Gilbert, C. Lemaréchal, C. Sagastizábal, Optimisation numérique (Numerical optimization), in: Aspects Théoriques et Pratiques (Theoretical and Applied Aspects), Mathématiques \& Applications, vol. 27, Springer, Berlin, 1997 (in French).

[9] F. Brezzi, On the existence uniqueness and approximation of saddle-point problems arising from Lagrange multipliers, RAIRO Anal. Numér. 8 (2) (1974) 129-151.

[10] F. Brezzi, M. Fortin, Mixed and Hybrid Finite Element Methods, Springer Series in Computational Mathematics, vol. 15, Springer, New York, 1991.

[11] P.-G. Ciarlet, The Finite Element Method for Elliptic Problems, North-Holland, Amsterdam, 1978.

[12] P. Coorevits, P. Hild, K. Lhalouani, T. Sassi, Mixed finite element method for unilateral problem: Convergence analysis and numerical studies, Math. Comp. 71 (2002) 237, 1-25.

[13] G. Duvaut, J.-L. Lions, Les inéquations en Mécanique et en Physique, Dunod, Paris, 1972.

[14] I. Ekeland, R. Temam, Analyse Convexe et Problèmes Variationnels, Collection Études Mathématiques, Dunod, GauthierVillars, Paris, 1974 (in French).

[15] R.S. Falk, Error estimates for the approximation of a class of variational inequalities, Math. Comp. 28 (1974) $963-971$.

[16] V. Girault, P.-A. Raviart, Finite Element Methods for Navier-Stokes Equations, Springer, Berlin, 1986.

[17] R. Glowinski, Numerical Methods for Nonlinear Variational Problems, Springer, New York, 1984.

[18] P. Grisvard, Elliptic Problems in Nonsmooth Domains, Monographs and Studies in Mathematics, vol. 24, Pitman, London, 1985.

[19] W. Han, B.D. Reddy, On the finite element method for mixed variational inequalities arising in elastoplasticity, SIAM J. Numer. Anal. 32 (1995) 1778-1807. 
[20] J. Haslinger, I. Hlaváček, J. Nečas, Numerical methods for unilateral problems in solid mechanics, in: P.G. Ciarlet, J.L. Lions (Eds.), Handbook of Numerical Analysis, vol. IV, Part 2, North-Holland, Amsterdam, 1996.

[21] P. Hild, Problèmes de contact unilatéral et maillages incompatibles, Thèse de l'Université Paul Sabatier, Toulouse 3, 1998.

[22] N. Kikuchi, J.T. Oden, Contact Problems in Elasticity: A Study of Variational Inequalities and Finite Element Methods, SIAM, Philadelphia, PA, 1988.

[23] J.-L. Lions, E. Magenes, Problèmes aux Limites non Homogènes, Dunod, Paris, 1968.

[24] E. Polak, G. Ribière, Note sur la convergence de méthodes de directions conjuguées, Rev. Française Informat. Recherche Opérationnelle 3 (1969) 35-43.

[25] Y. Renard, GETFEM ${ }^{++}, \mathrm{a} \mathrm{C}^{++}$package for finite element computations, http://www.gmm.insa-toulouse.fr/getfem (freely distributed).

[26] L. Slimane, Méthodes mixtes et traitement du verrouillage numérique pour la résolution des inéquations variationnelles, Thèse l'Institut National des Sciences Appliquées de Toulouse, 2001.

[27] L. Slimane, A. Bendali, P. Laborde, Mixed formulations for a class of variational inequalities, Modél. Math. Anal. Numér. 38 (1) (2004) 177-201.

[28] M. Suri, Analytic and computational assessment of locking in the $h p$-finite element, Comput. Methods Appl. Mech. Engrg. 133 (1996) 347-371. 